\title{
Le fil de la pensée tisserande
}

«Affordances » de la matière et des corps dans le tissage

\section{Myriem Naji}

\section{(2) OpenEdition}

\section{Journals}

Édition électronique

URL : https://journals.openedition.org/tc/4826

DOI : $10.4000 /$ tc. 4826

ISSN : 1952-420X

Éditeur

Éditions de l'EHESS

\section{Édition imprimée}

Date de publication : 1 décembre 2009

Pagination : 68-89

ISBN : 978-2-7351-1301-9

ISSN : 0248-6016

Référence électronique

Myriem Naji, «Le fil de la pensée tisserande », Techniques \& Culture [En ligne], 52-53 | 2009, mis en ligne le 01 août 2012, consulté le 29 septembre 2022. URL : http://journals.openedition.org/tc/4826 ; DOI : https://doi.org/10.4000/tc.4826

Ce document a été généré automatiquement le 29 septembre 2022.

Tous droits réservés 


\section{Le fil de la pensée tisserande}

«Affordances » de la matière et des corps dans le tissage

\section{Myriem Naji}

\section{NOTE DE L'ÉDITEUR}

Le tissage appartient au domaine des mathématiques. Une approche par l'embodiment ou la corporéité permet de rendre compte de la manière dont les tisseuses du Sirwa utilisent leurs expériences et capacités sensorimotrices en combinaison avec les affordances matérielles et culturelles de cette technique pour construire des concepts mathématiques.

Dans les montagnes berbères du Sirwa dans l'Anti-Atlas marocain, la quasi-totalité des foyers produisent des tapis pour le marché international toute l'année. Les femmes tissent collectivement, dans l'espace domestique, entre femmes de la même maisonnée et du voisinage. L'une des questions qui m'a interpellée tout au long de mon terrain a été celle de comprendre comment les tisseuses parviennent à produire des tapis symétriques sans l'usage de support cognitif tel qu'un dessin comme cela est fréquemment pratiqué dans les ateliers de tissage urbains au Maroc. En effet, le tissage sur métier vertical implique une progression vers le haut qui cache la majeure partie du travail à l'ouvrière. Ni les réponses des tisseuses ni l'observation attentive de leur pratique ne suffisaient à résoudre ces questions. Mon propre apprentissage et la notion d'affordance (Gibson 1979 ; Knappett 2004 ; Norman 1999) ainsi que la perspective des théories de l'action située et de la cognition incarnée (Johnson 1987 ; 1999 ; Kirsch 1995 ; Lakoff \& Johnson 1980 ; Keller \& Keller 1996 ; Lave 1988 ; Lave \& Wenger 1991 ; Varela \& al. 1991) m'ont aidée à ébaucher quelques hypothèses sur la manière dont les tisseuses construisent des concepts géométriques et mathématiques à partir de l'expérience sensorimotrice du tissage. Les théories de l'action/cognition située et incarnée ou de l'inscription corporelle de l'esprit (Quéré 1997) postulent que l'esprit n'est pas désincarné mais est situé dans un corps, lui-même situé dans un environnement social et matériel. Cette approche subjective et corporelle du sujet en action a l'avantage de coupler les expériences et capacités sensorimotrices humaines 
avec un contexte culturel et physique donné. Par affordance, j'entends les propriétés de l'environnement qui ouvrent des possibilités d'action, de perception et de pensée au sujet. Mon utilisation de la notion d'affordance englobe, j'y reviendrai, le couplage sujetenvironnement (matériel et social) et tient compte des " propriétés » de la matière aussi bien que de celle (du corps) du sujet.

\section{Spécificité de la technique du tissage et affordances}

Ce qui fait la spécificité de la technique du tissage dans le Sirwa, ce sont ses affordances (les possibilités d'action) qu'offre ce dispositif technique et ses limites et contraintes. Alors que le métier à tisser horizontal (masculin) utilisé par le darrâz en ville ne permet de confectionner que des tissus, le métier vertical (Fig. 1) sert aussi à produire des tapis. Sa simplicité est proportionnelle à la complexité et la variété des techniques auxquelles ce dispositif se prête. 

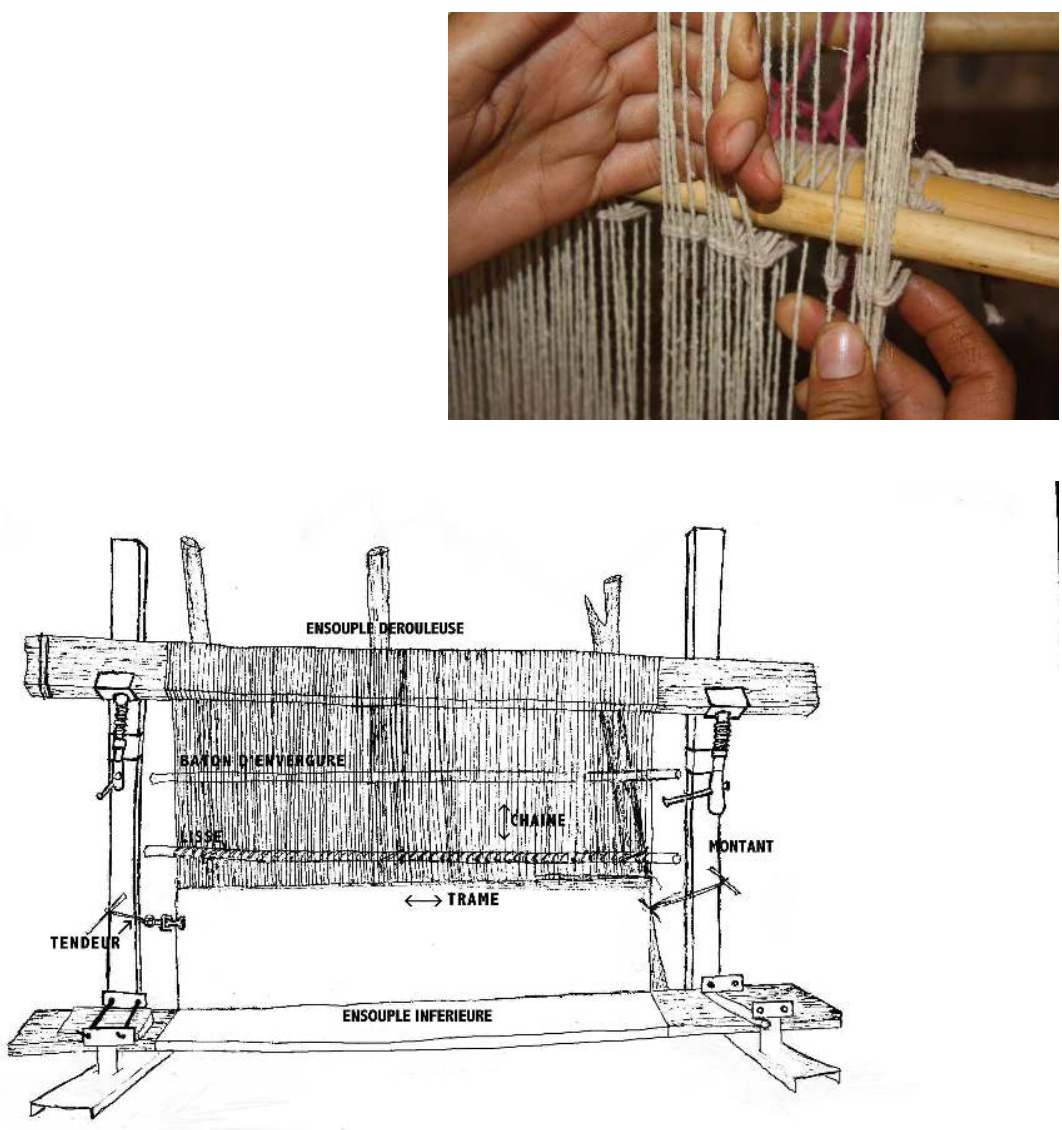

(CMyriem Naji

\section{Le métier à tisser vertical}

Aujourd'hui les montants des métiers sont en fer, ce qui signifie qu'une main-d'œuvre moindre est nécessaire pour les opérations d'enroulement du tissu et de déroulement de la chaîne. Avec un instrument plus stable et rigide, les tapis ne « gondolent » plus et leur largeur est constante d'une extrémité à l'autre. Les ensouples (ou poutres) supérieures et inférieures toujours confectionnées en bois mais beaucoup plus longues qu'autrefois sont adaptées à la demande de tapis plus larges. Un système d'écrou permet de baisser tous les deux jours environ la poutre supérieure pour libérer plus de chaîne tandis que la partie tissée est enroulée autour de la poutre inférieure. La tension des fils de la chaîne est accentuée par l'usage de larges branches (azrâz) qui tirent sur la chaînette de la lisse (nouées sur un roseau, cf. Fig. 6). (Fig. 1)

1 Le tissage certes imite les matières que sont la laine et le cuir puisque cette technique produit un entrelacement de couches fibreuses animales, mais elle offre aussi la possibilité de créer des épaisseurs et des formes fort variées. Tout se passe comme si le nœud, en tant que technique qui couvre et encercle, rendait l'usage de l'aiguille superflu et allait de pair avec la souplesse des objets tissés que l'on peut enrouler autour du corps, sur d'autres objets ou sur eux-mêmes (par exemple en baluchon).

2 Le tissage sur métier vertical est aussi caractérisé par trois principales contraintes matérielles : à la différence de la peinture et de la broderie où les motifs sont ajoutés 
sur une surface, dans la confection des tapis, les motifs et le fonds sont construits ensemble par l'entrecroisement des fils de la chaîne et de la trame.

3 Une autre particularité du tissage vertical est que cette activité impose une progression verticale du travail du bas vers le haut, exigeant des tisseuses qu'elles s'y adaptent physiquement. Elles se rehaussent par des coussins au fur et à mesure que le tissage croît en taille (Fig. 2 a).

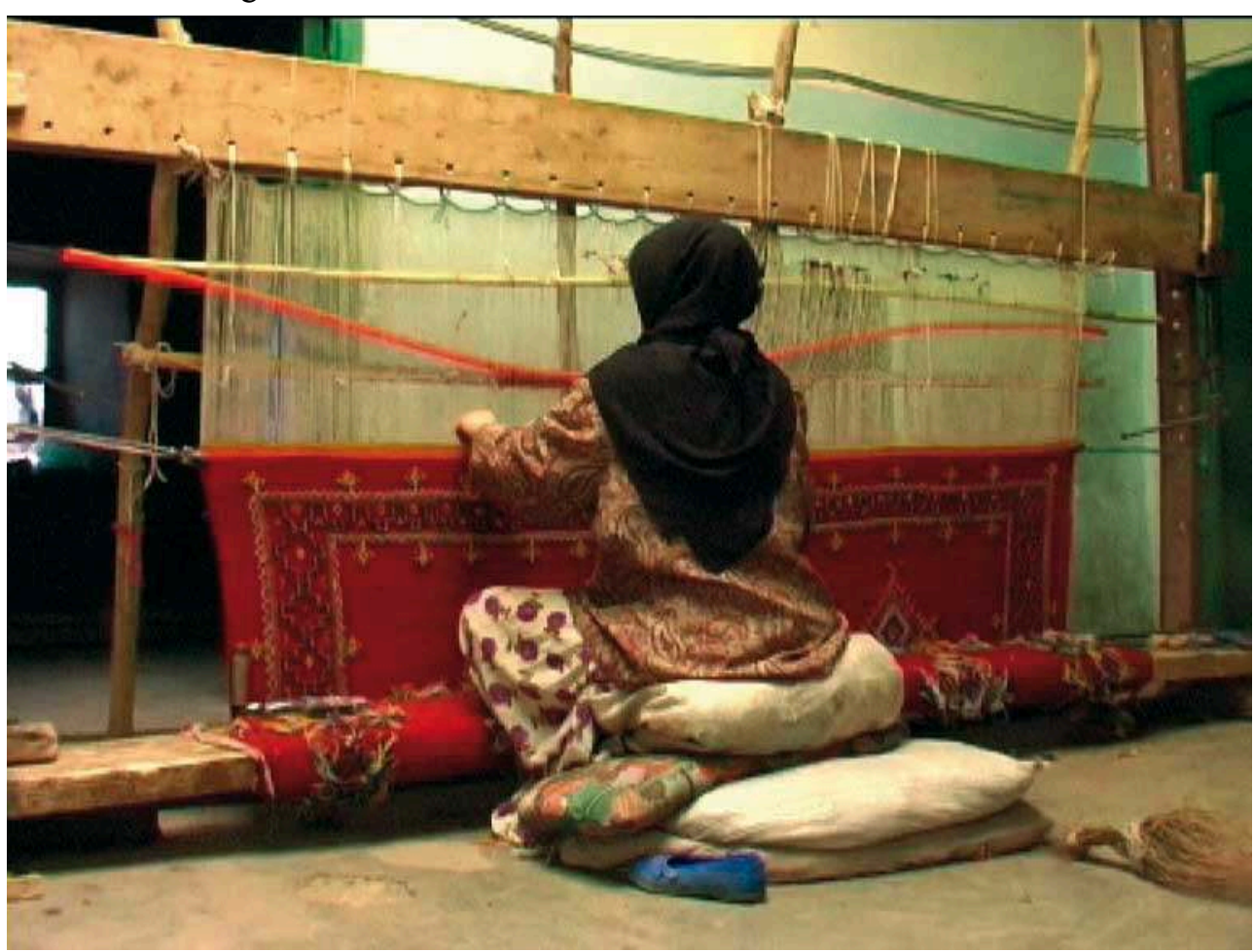

(CMyriem Naji

\section{Adaptation du corps à la progression du tapis}

Le tapis est quasiment fini. Une retardataire se retrouve seule ; elle s'est rehaussée grâce à plusieurs coussins ou sacs et doit se ternir toute droite pour pouvoir atteindre son ouvrage. (Fig. 2 a)

4 Une fois atteinte une certaine hauteur à laquelle élever les bras de manière prolongée devient douloureux et dans laquelle le passage des duites si près du rang de lisses leur écorche les mains, il devient alors nécessaire de libérer la partie non tissée de la chaîne et d'enrouler la partie travaillée autour de la poutre inférieure (Fig. 3) ; les tisseuses doivent alors se courber en deux pour pouvoir travailler très bas (Fig. 2 b). 


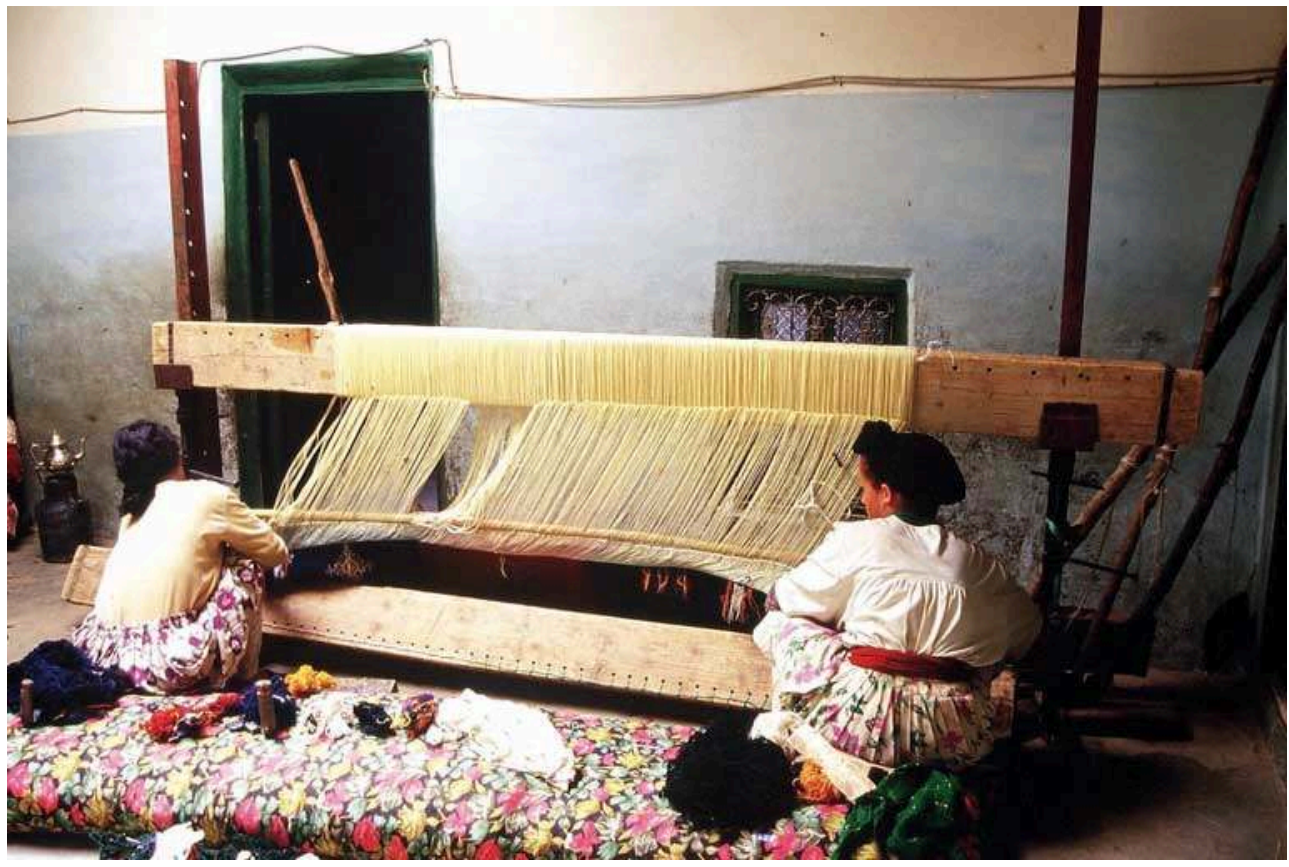

(CMyriem Naji

\section{Enroulement de la partie tissée autour de l'ensouple inférieure}

Les écrous ont été dévissés, la poutre supérieure baissée et déroulée et les jeunes filles sont à présent en train de retourner l'ensouple inférieure sur elle-même. II faudra ensuite remonter l'ensouple supérieure, et renforcer la tension de la chaîne en rattachant la barre d'écartement aux trois branches (azrâz) à l'arrière du métier. (Fig. 3)

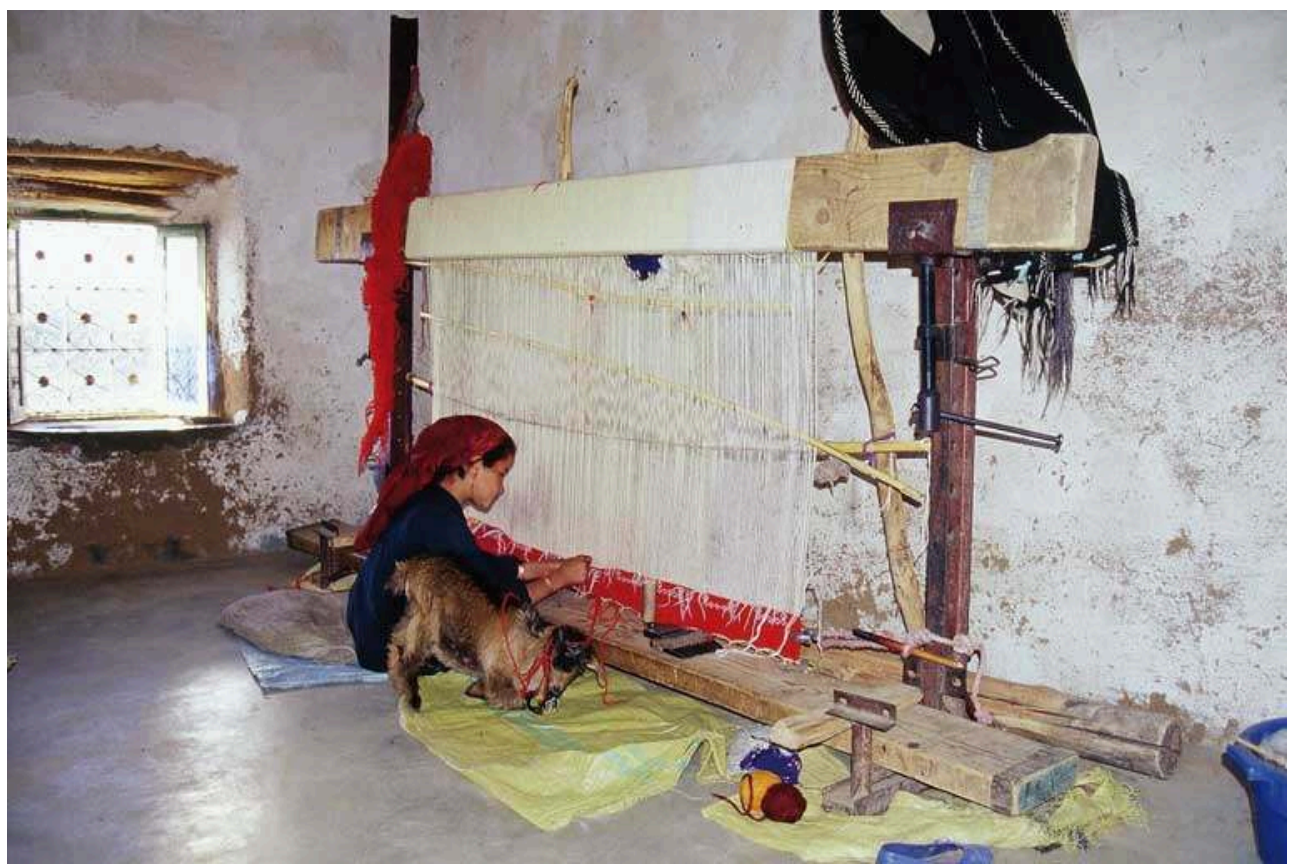

(CMyriem Naji

\section{Fillette courbée}

Par contraste avec la tisseuse de la figure 2 a, cette petite fille doit se courber pour pouvoir travailler sur le tapis, qui venant d'être commencé, se trouve au niveau de son giron. La chèvre naine à ses côtés est un animal familier qui côtoie au quotidien les femmes de la maison, nettoyant le sol et parfois dormant avec la maîtresse de maison. (Fig. 2 b) 
Il en résulte, ce qui a des conséquences sur la cognition, que les tisseuses tout au long de leur travail ne voient qu'une infime portion du tissage, le restant étant caché dans l'ensouple inférieure. Ces contraintes signifient qu'il est impossible de placer d'abord le motif central et ensuite les autres, et qu'il faut d'une part penser de manière ascendante et d'autre part trouver des moyens pour pallier l'invisibilité de la plus grande part de l'ouvrage.

6 À cela s'ajoute une exigence culturelle : la nécessité de produire des tapis symétriques, l'absence de symétrie étant interprétée comme une preuve de maladresse et d'ignorance. Cette symétrie touche aussi bien les motifs que la composition : il s'agit de la reproduction par réflexion de tous les éléments de la composition dans les deux moitiés du tapis séparées par un motif central, lui-même symétrique (Fig. 4).

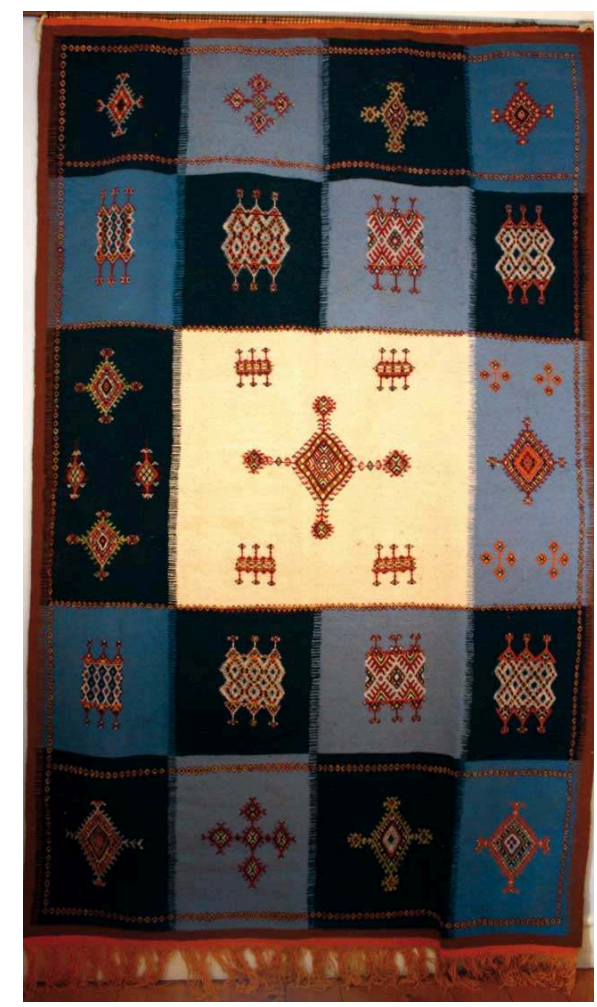

(C)Myriem Naji

Tapis symétrique en échiquier comportant une grande variété de rhombes

Les couleurs du fond, peu usuelles, ont été choisies par l'auteure. (Fig. 4)

\section{Incorporation et affordance matérielles, cognitives et corporelles}

J'ai montré ailleurs que la pratique du tissage implique un dispositif plus large que le métier à tisser. Le terme vernaculaire berbère astta ne se limite pas à cet instrument mais désigne à la fois l'activité, l'objet en devenir et l'espace (physique et social) où se déroule l'action. La notion d'affordance et la perspective de l'incorporation (Rosselin 2006) permettent d'analyser le processus d'apprentissage par lequel le sujet et le dispositif technique forment un couplage inséparable. 
7 En liant les propriétés d'un objet, une matière ou une substance aux actions efficaces que ceux-ci renferment, Leroi-Gourhan $(1943,1945)$ a anticipé les découvertes des psychologues (Gibson 1979) et des chercheurs en neurosciences. Jeannerod (1994) propose que les caractéristiques des objets activent certains schémas moteurs prédéterminés, autrement dit que les objets peuvent déclencher certaines actions. Cela est confirmé par la découverte des neurones canoniques : la simple observation d'un objet impliquerait au niveau neurophysiologique une simulation inconsciente de l'action qu'il renferme (Gallese 2000 a).

Cependant, la notion d'affordance que j'utilise ici ne décrit pas seulement la perception des propriétés de l'environnement matériel et social par le sujet; elle tient aussi compte des propriétés de son propre corps. Comme l'ont souligné Merleau-Ponty (1945) et Gibson (1979), percevoir le monde, c'est se percevoir soi-même en mouvement dans cet environnement. En manipulant la laine, les tisseuses ne perçoivent pas seulement les potentialités que cette matière leur ouvre, elles le font à partir d'une structure anatomique et de capacités sensorimotrices particulières, elles-mêmes en partie culturellement construites, et par le biais du schéma corporel. Celui-ci ou image du corps, est une construction neurophysiologique et symbolique qui se réfère à la sensation que l'on a de notre position et de nos mouvements. Les praticiennes agissent sur la matière en même temps qu'elles sont " agies " à travers la confrontation de deux résistances ou matérialités : celle de astta et celle de leur corps (Naji 2009). La compétence et les gestes techniques efficaces émergent d'un long travail d'incorporation d'astta dans leur schéma corporel ; apprentissage au terme duquel la matérialité n'est plus perçue comme extérieure au corps dont la limite serait la peau, la main ou la partie du corps en contact avec la matière. Cette incorporation mobilise tous les sens à des degrés variables selon les différentes étapes de la chaîne opératoire et la compétence des tisseuses. La résistance de l'ensemble des fils de la chaîne est ainsi perçue durant le tissage visuellement, tactilement et kinesthésiquement, par le biais du peigne à tasser, de sons métalliques et sourds et de vibrations ressenties dans les genoux, voire les dents. Le dispositif technique de tissage se rapporte donc au couplage femme-astta. Il implique des contraintes et des affordances qui sont des propriétés de la matière aussi bien que du sujet.

\section{Appréhension de la matière et des amordances du dispositif technique}

L'apprentissage par échafaudage (Childs \& Greenfield 1980) correspond à une initiation progressive au travail de la laine en commençant par les opérations les plus simples. Le filage (de moins en moins pratiqué) est appris avant le tissage. Ce premier contact physique avec la matière fibreuse, en tordant et étirant la laine en un fil plus ou moins fin et résistant, permet une prise de conscience de sa plasticité. Certes le fil autorise la tension et la ligne droite, mais il se prête aussi à être enroulé en pelote, replié sur luimême ou autour d'un autre fil pour former un nœud. D'une matérialité semi-plastique (Balfet \& Desrosiers 1987 ; Leroi-Gourhan 1943) fine et longue, les fils tracent des lignes qui se croisent obliquement ou à angle droit, et qui superposées forment des couches parallèles. Comme les cheveux que l'on tresse ou tord ${ }^{1}$, les fils s'emmêlent mais ils offrent aussi la possibilité d'être organisés pour créer une forme ordonnée. Aussi, durant les opérations antérieures à la confection du rang de lisses (inliten, Fig. $5 \mathrm{a}$ et b), 
qui alors organise les fils de la chaîne de manière permanente, un grand soin est pris afin que ces fils ne s'emmêlent pas, en particulier durant le montage de la chaîne sur les ensouples (Fig. 6).
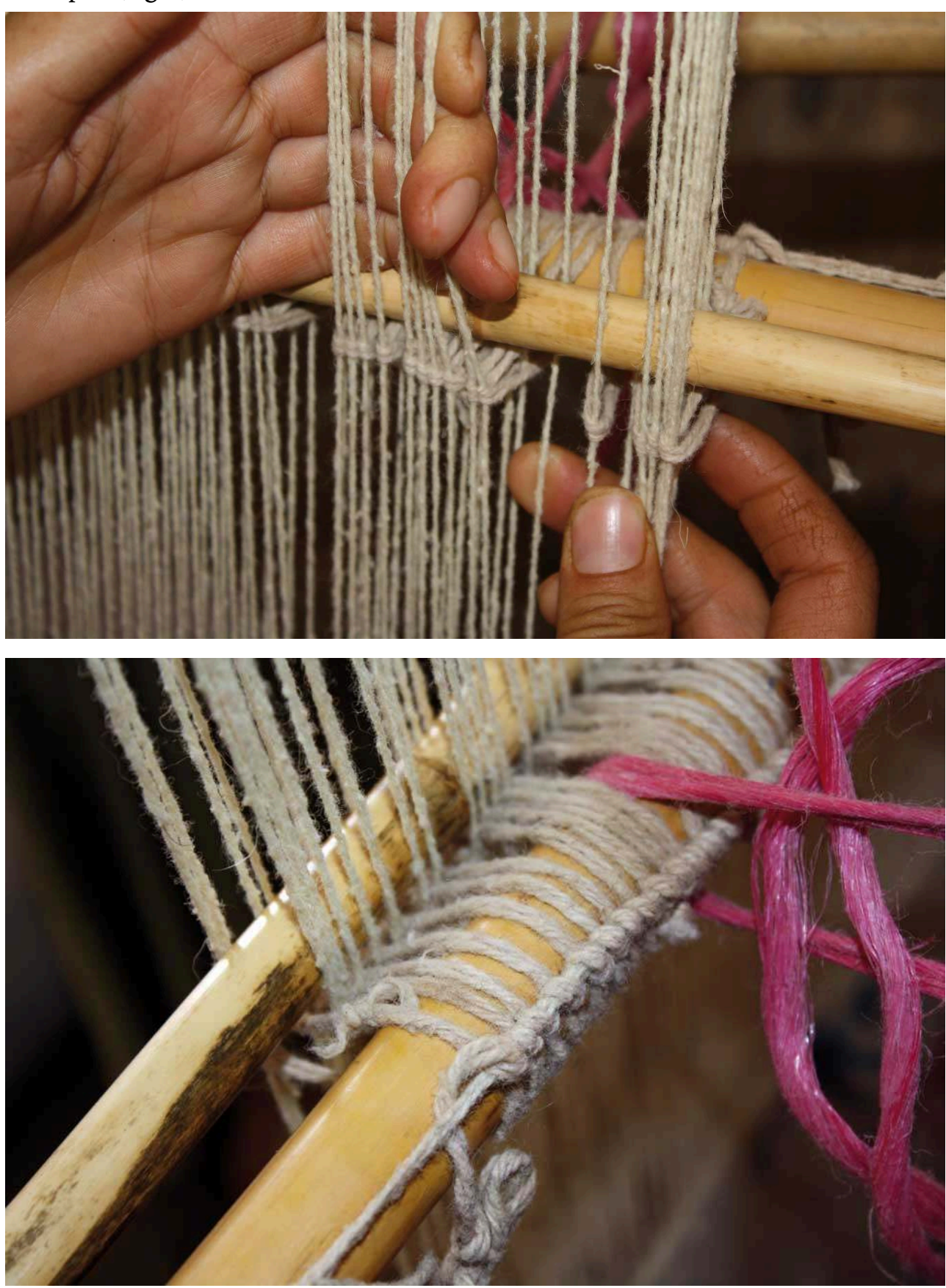

(C)Myriem Naji

Rang de lisses (inliten) vue de face et de dos

9 Face : La barre d'écartement est baissée, ce qui permet à Ijja de tirer sur cette nappe de fils de la chaine et d'y passer sa main gauche. Lorsqu'elle remontera la barre ce sont les fils de l'arrière qui passeront devant.

Dos : alors que de face, on voit le système de nœuds coulants qui retient de manière plus ou moins lâche les fils de chaîne, de dos c'est la chaînette du inliten qui est visible. 
Le fil rose est attaché à une des grandes branches (azrâz) qui maintiennent la tension de la chaîne. (Fig. 5 a et 5 b)

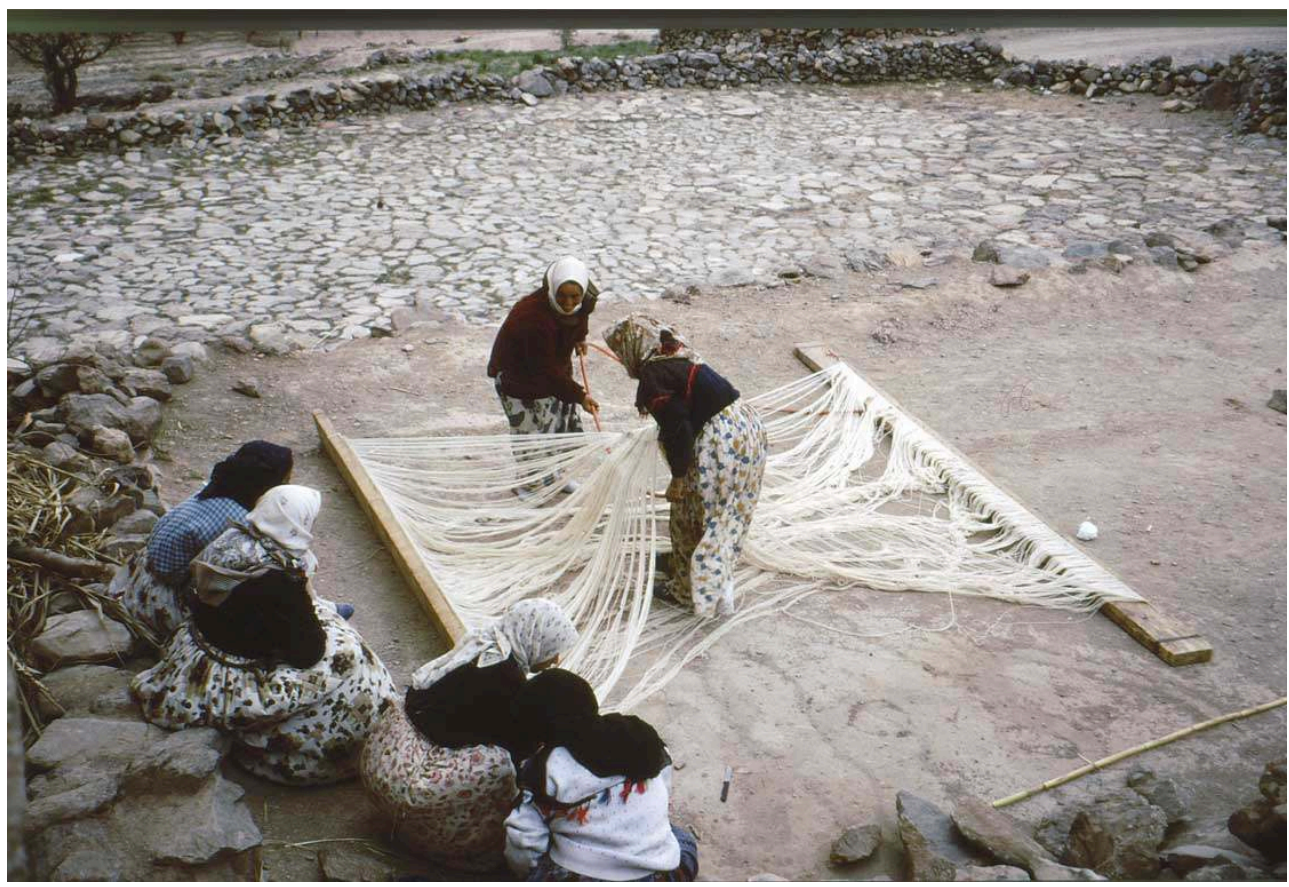

Fixage de la chaîne sur les ensouples

Par un matin d'hiver, sur une aire à battre, après que les femmes adultes aient fini d'ourdir la chaîne, les jeunes filles ont déposé les deux ensouples de manière parallèle afin de pouvoir y coudre les deux extrémités (tigrut, cf. fig. 13) de la chaîne. Avant d'enrouler la chaîne autour de l'ensouple supérieure, elles glissent entre les fils de chaîne, une barre d'écartement plus flexible que le roseau : un tuyau orange. Les risques d'emmêlement sont très visibles. (Fig. 6)

11 Grâce à la pratique du tissage précédant la confection de l'inliten, les jeunes filles développent une compréhension de ces mécanismes du tissage, à force de monter et descendre la barre d'écartement qui écarte et resserre alternativement les nappes de fils, et de passer leurs mains entre celles-ci. Les nœuds coulants de l'inliten, en retenant un fil de chaîne sur deux, modifient légèrement leur trajectoire sans leur ôter leur dynamisme, créant ces nappes de fils parallèles mais alternativement ouvertes et fermées. Le nœud fournit ainsi non pas une surface mais un espace multidimensionnel. Pour rendre la tâche moins lassante et ardue, mais probablement aussi pour stimuler l'émulation, cette opération est effectuée par deux personnes, chacune commençant à former la chaîne de l'inlinten depuis l'une des lisières pour se retrouver au centre du métier à tisser.

12 La participation à tous les travaux précédant le tissage imprègne donc les jeunes filles des propriétés de la matière et les familiarise aux gestes techniques. Ainsi, alors qu'il est rare qu'elles apprennent à nouer la chaînette (tigrut) qui constituera la largeur du tapis, on leur assigne toujours le rôle moins expert de débobiner la pelote dont le fil est passé à l'une puis à l'autre ourdisseuse (Fig. 7). 


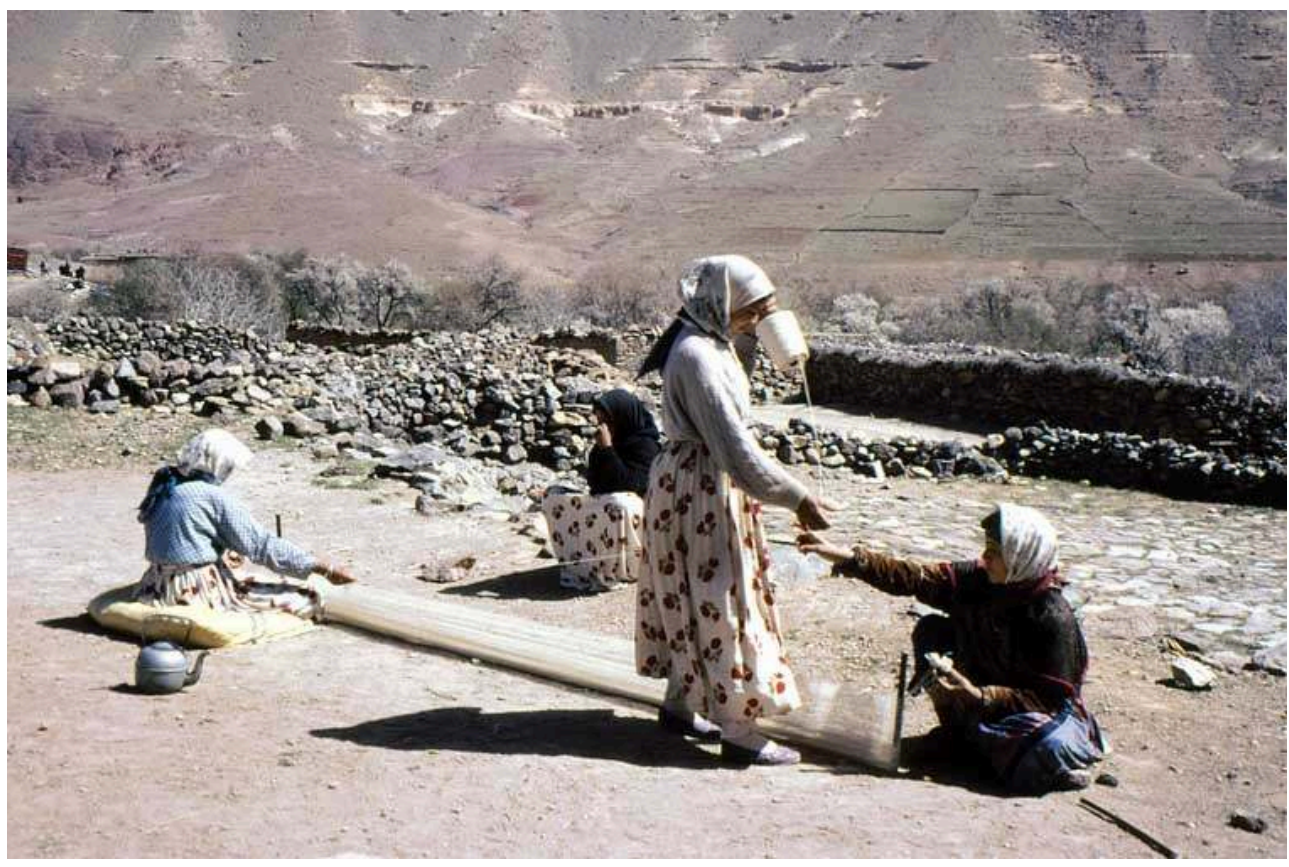

(C)Myriem Naji

Opération d'ourdissage

Elle nécessite trois personnes : deux assises devant un piquet reçoivent de la troisième qui va et vient entre elles un fil qu'elles nouent entre deux fils doublés formant une chaînette (tigrut) autour du piquet (cf. Fig. 13).(Fig. 7)

13 Cependant, la compréhension de la tension de la chaîne ne signifie pas pour autant l'investissement immédiat de toutes ses potentialités : ces affordances émergent par la pratique dans un contexte socioculturel particulier. Ainsi, il m'a fallu un certain temps pour comprendre que les gestes de brochage les plus efficaces consistaient non pas à manipuler les fils indépendants, mobiles et lâches autour des fils de chaîne tendus, mais au contraire à faire avancer ces derniers pour y accrocher les premiers. Ma perception de la chaîne comme un écran fixe et rigide m'a empêchée d'envisager ses qualités élastiques. C'est cette tension plus ou moins forte et admettant une déviance par rapport à la ligne droite, qui justement rend la manipulation de la chaîne plus économique et fait qu'elle constitue un espace dynamique multidimensionnel.

14 Une anecdote nous éclaire sur le rôle attribué localement à cette imprégnation sensorimotrice dans l'apprentissage. Un jour, pour mon bénéfice, les jeunes filles de la famille Ayt 'Abdallah mirent les fillettes de la maison, âgées de douze et quatorze ans, devant le métier et, sans ambages ni préparation, leur ordonnèrent de passer les trames et de les tasser. La valeur informative de cet événement complètement artificiel réside dans le fait que tout se passa comme si les sœurs aînées considéraient que l'observation prolongée à laquelle les fillettes avaient été exposées en tant que membres féminins de la famille constituait une initiation suffisante. Houspillées à chaque fois qu'elles faisaient mine de ralentir, les fillettes n'avaient ni le temps de réfléchir avant d'agir ni celui d'analyser leurs mouvements. On encourageait l'aspect réactif de la perception et de l'action. La compétence revient donc à être capable d'appréhender rapidement et efficacement un répertoire d'actions potentielles que la 
matière renferme et que la culture dicte. La plus lente des deux fillettes à acquérir ces automatismes fut automatiquement qualifiée de moins intelligente.

\section{Penser avec le corps des autres}

Il existe des moments d'intimité personnelle où la tisseuse trouve la solution à un problème et construit son savoir seule dans son corps à corps avec la matière. Pour comprendre comment une forme a été produite, Asia, une tisseuse débutante, ne se contente pas d'imiter les gestes de Huda, plus experte qu'elle. Elle défait les fils attachés par Huda et essaie de les replacer au bon endroit. Avec le temps, la répétition des gestes correspondant à des formes particulières entraîne une articulation fluide des représentations visuelles et des représentations motrices qui assure une mémorisation telle que la simple vue d'un motif permet sa reproduction matérielle ultérieure. Mais l'imprégnation sensorimotrice des propriétés de l'environnement se fait aussi à travers le corps des autres. Les jeunes filles apprennent à tisser sur le même tapis avec une tisseuse plus compétente, soit leur mère, soit une sœur ou cousine. Au terme de deux ans de pratique quasi quotidienne, une tisseuse est considérée comme experte. Le tissage, comme d'autres activités domestiques, constitue la matière sur laquelle les liens entre les femmes et la transmission du savoir s'effectuent. C'est dans leurs familles qu'elles apprennent ce qu'est un bon travail, c'est-à-dire à évaluer la qualité de leur ouvrage par rapport aux autres tisseuses du village. Dans un contexte où l'activité du tissage n'est pas toujours considérée de gaieté de cœur, la concurrence entre les tisseuses (et leurs familles) en termes de statut social constitue une motivation importante. En effet, elles se distinguent à travers leurs compétences techniques et la visibilité de leur contribution au revenu de la famille.

En travaillant avec une autre sur le même objet en devenir, l'apprentie appréhende la matière en se positionnant de la même manière et en adoptant la même perspective que celle qui est assise à ses côtés, et ce sans avoir à effectuer une transposition spatiale extrême. Les neurones miroirs qui interviennent dans le contrôle de l'action, dans la simulation et dans l'expérience des actions, émotions et sensations sont actifs aussi quand nous sommes les témoins de celles-ci chez les autres (Gallese 2000 b).

Pareillement à ces maçons maliens, étudiés par Marchand (2007) qui partagent une histoire de travail commune, les tisseuses construisent ensemble, simultanément, des représentations (visuelles, verbales, motrices) de ce qu'elles veulent accomplir et de ce qu'est un bon travail. Le processus d'imitation, qui implique la production d'une approximation individuelle et pas exactement identique aux représentations de l'experte, porte donc en lui les germes du changement. Les tisseuses articulent ces représentations dans leurs performances motrices pour coordonner leur travail parfois sans échange verbal, comme quand le peigne à tasser et les écheveaux passent d'un giron à l'autre ; il n'est pas non plus nécessaire de se parler pour savoir comment et où la jonction entre les deux parties respectives sur lesquelles les tisseuses travaillent doit se faire. Dans ce contexte de pratique collective, il y a toujours une personne plus expérimentée sur laquelle on peut compter. Par exemple, connaissant les préférences esthétiques de chaque tisseuse, la plus experte des tisseuses est capable de les aider à reconstituer un motif. Les noms des motifs ou de certains de leurs éléments ont une valeur mnémotechnique et intersubjective de communication. 
17 À cette lecture commune des actions potentielles présentes dans la matière agie se mêlent indissociablement des conventions culturelles et familiales, à valeur morale. Ainsi en laissant pendre la trame en escalier (isghayn, Fig. 9 b et 11) de manière à ce que sa voisine puisse prendre la suite quand elle le désire, la tisseuse sait que cela affectera la régularité des duites d'une lisière à l'autre. Les tisseuses perfectionnistes rejettent ce procédé technique (isg) et se distinguent ainsi de celles qui le pratiquent.

\section{Le concept « fil-ligne »}

Si les affordances et contraintes jouent un rôle crucial dans le raisonnement et la conceptualisation, je me concentre dans cet article sur un élément de cette matérialité qui me semble primordial pour la cognition, le fil, parce qu'il est à la base de la formation de ce que je nomme le concept de « ligne » ou de « ligne-fil ». Ce concept n'est pas émique mais correspond à mon interprétation de ce phénomène d'incorporation par lequel l'engagement physique répété des tisseuses avec la matérialité la plus élémentaire d'astta ${ }^{2}$ engendre la formation de concepts complexes. Le concept fil-ligne constitue le fondement de la pensée tisserande. À la fois objet matériel et concept, cette forme géométrique élémentaire est manipulée et placée par rapport à d'autres lignes pour créer et penser d'autres concepts plus sophistiqués : polygones à trois ou quatre côtés, dont le rhombe (<>), constitué de deux triangles congruents séparés par une diagonale qui constitue un axe de symétrie en réflexion ou miroir (Fig. 8).

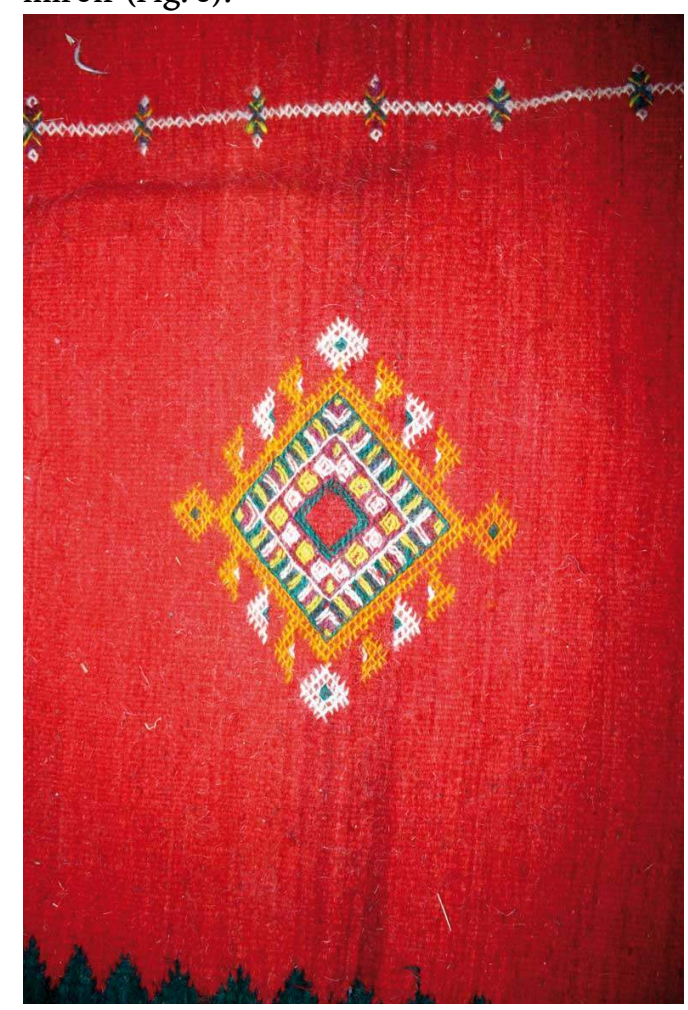

(CMyriem Naji 


\section{Symétrie en réflexion ou miroir}

Exemple de motif symétrique basé sur le rhombe. Fadma était très fière de sa nouvelle prouesse technique. En commençant par le rhombe orange du bas, elle a réussi à faire en sorte que le rhombe blanc succède avec harmonie à un triangle orange, suivi d'un triangle blanc et d'un autre orange. (Fig. 8)

Les formes expérimentées par l'expérience sensorimotrice deviennent des entités manipulables mentalement. Les tisseuses ont donné des noms aux concepts élaborés et aux motifs qui leur permettent de les classer et de les comparer dans les moments de réflexion personnelle, mais aussi d'en discuter avec d'autres tisseuses.

Un autre élément important de la pensée spatiale tisserande est le schéma " image ». Selon Lakoff et Johnson (1980; Johnson 1987), nos concepts émergent de nos expériences inconscientes sensorimotrices de tous les jours et reposent sur notre infrastructure biologique. C'est parce que nous avons un corps capable de se déplacer sur deux jambes dans une position verticale et que nous avons des organes perceptuels qui peuvent repérer des objets en mouvement que nous avons développé des schémas images. Ceux-ci ne sont pas des images mentales, mais des structures dynamiques et récurrentes qui organisent nos représentations mentales, et donnent cohérence et sens à nos expériences perceptuelles préconceptuelles, non-formelles et nonpropositionnelles (Johnson 1987, 1999). Ainsi, le sens de l'équilibre est inscrit dans notre position verticale. Il donne sens à d'autres expériences corporelles comme celle des quantités (trop ou pas assez) et du déséquilibre. Johnson et Lakoff identifient plusieurs schémas, parmi lesquels je retiendrai les suivants : la verticalité, l'équilibre, le récipient (lié à des concepts comme " dans " et " hors ", et à la perception du corps et des objets comme des récipients) et le schéma source-chemin-but (de ... à). Ces schémas images, nous allons le voir, jouent un rôle important dans l'émergence de concepts géométriques et mathématiques liés à l'organisation spatiale du tissage.

Je commencerai en décrivant comment la perception kinesthésique du concept de ligne par les tisseuses s'effectue en symbiose avec celui de la gravité et de l'équilibre de leur propre corps. Assises droites devant l'astta, les tisseuses suivent le tissage vers le haut. Lorsqu'elles tassent ou passent la trame, elles se meuvent d'avant en arrière et obliquement de droite et à gauche (et vice versa) dans la limite de leur assise et donc de l'équilibre. La verticalité et l'horizontalité sont aussi des caractéristiques de l'instrument technique et de l'objet tissé. Le métier à tisser (Fig.1) est un cadre constitué de lignes verticales et horizontales : la chaîne est formée de fils droits parallèles tendus verticalement entre deux poutres horizontales, elles-mêmes soutenues par des montants verticaux. La trame qui la rencontre à angle droit est 
constituée de lignes horizontales superposées et plus lâches qui sont passées transversalement (Fig. 9 a, 9 b).

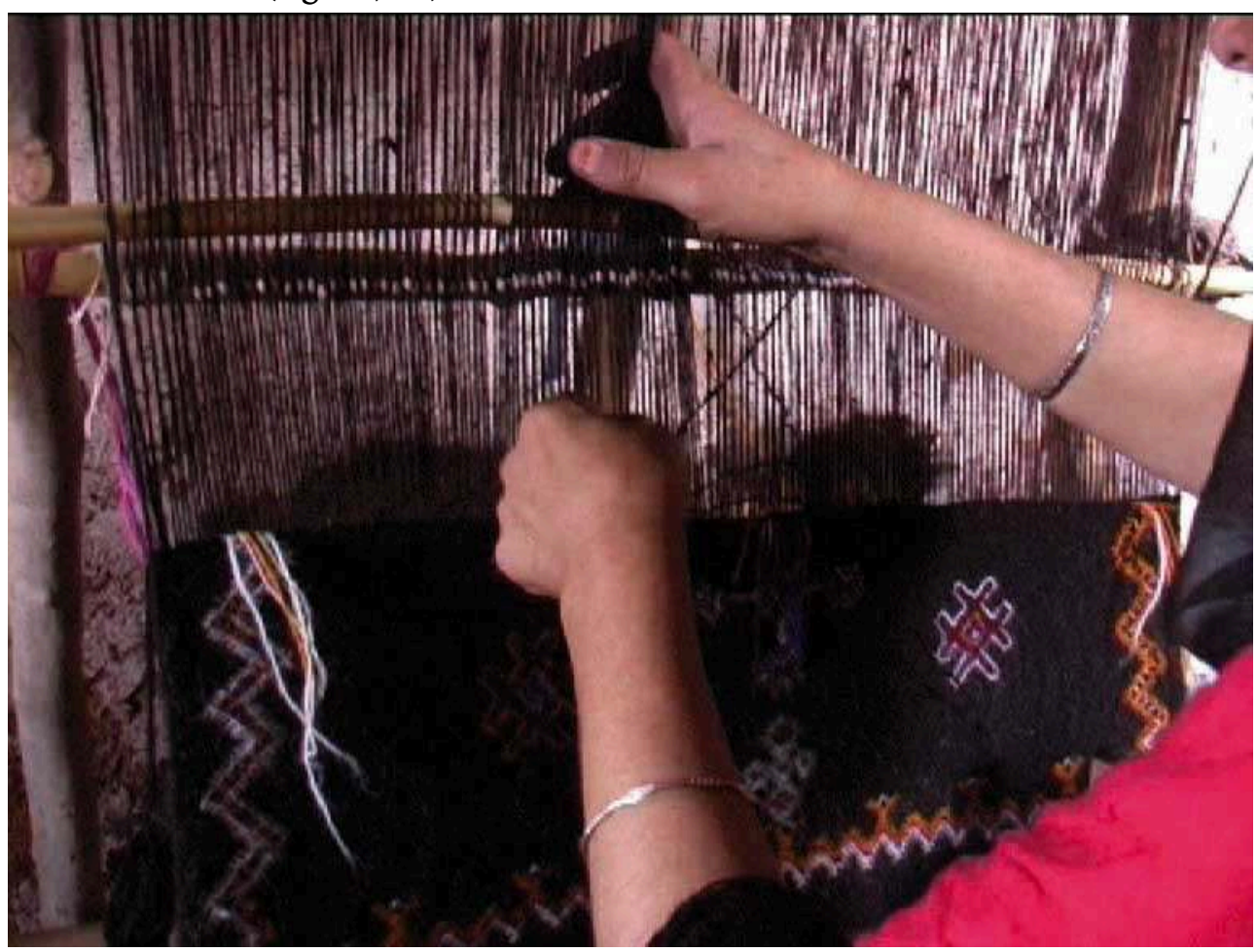

(C)Mriem Naji

\section{Passage des fils de trames entre les nappes de la chaîne}

Sous la main droite de la tisseuse au pull rose on peut voir le isghayn, sorte d'escalier qui permet de tisser sur la partie devant soi sans se préoccuper du rythme des autres tisseuses. (Fig. 9 a et 9 b) 


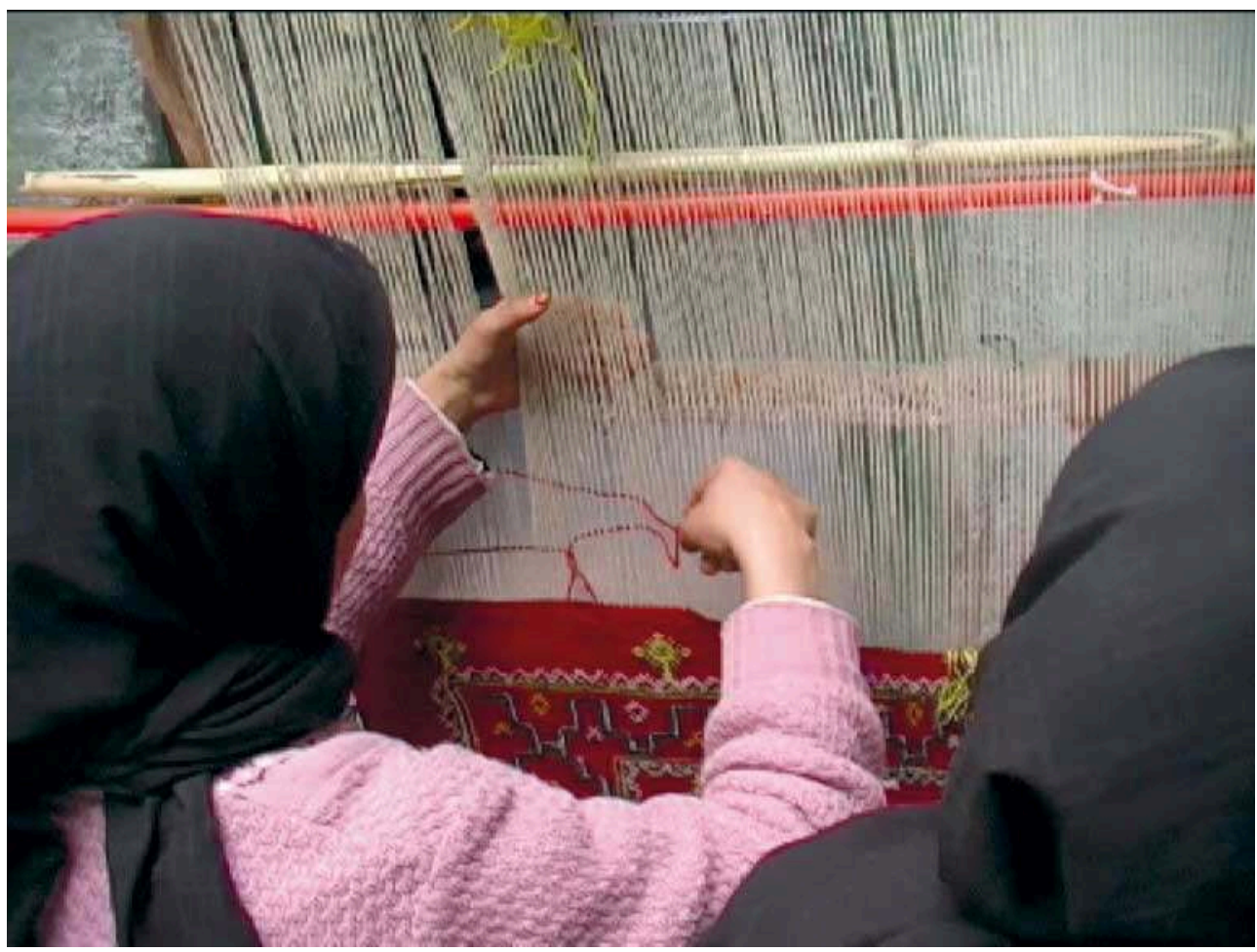

(C)Myriem Naji

\section{Passage des fils de trames entre les nappes de la chaîne}

Sous la main droite de la tisseuse au pull rose on peut voir le isghayn, sorte d'escalier qui permet de tisser sur la partie devant soi sans se préoccuper du rythme des autres tisseuses. (Fig. 9 a et 9 b)

21 Sur ces formes géométriques élémentaires verticales et horizontales, les fils des motifs brochés ne tiennent qu'obliquement : attachés à certains fils de la chaîne, ils sont retenus par les fils de trame au fur et à mesure qu'ils se meuvent vers le haut (Fig. $10 \mathrm{a}$, $10 \mathrm{~b}, 10 \mathrm{c}, 10 \mathrm{~d}$, fig. 11). 


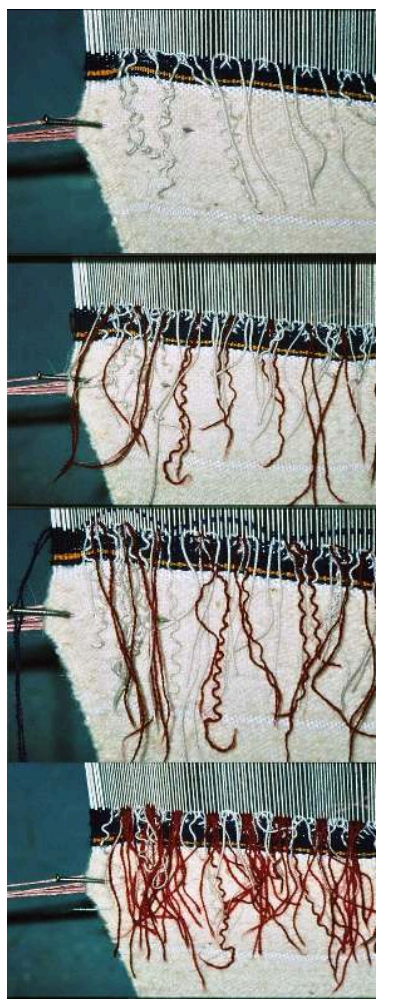

(C)Myriem Naji

Progression des motifs brochés après chaque passage de duite (bleu marine)

Des fils indépendants blancs ont été placés à intervalle régulier au-dessus de la trame bleue. Ils sont maintenus par un passage de duite, puis sont entrecroisés entre eux pour former une sorte de $\mathrm{x}$. Nouveau passage de duite, cette fois on ajoute d'autres fils indépendants blancs puis après un autre passage de duite des fils indépendants rouges. À gauche, on peut voir un tendeur des plus frustres: un long clou dont la pointe est fichée dans le tissu et dont l'autre extrémité est attachée par un cordon rose au montant du métier. (Fig. 10 a, 10 b, 10 c, 10 d

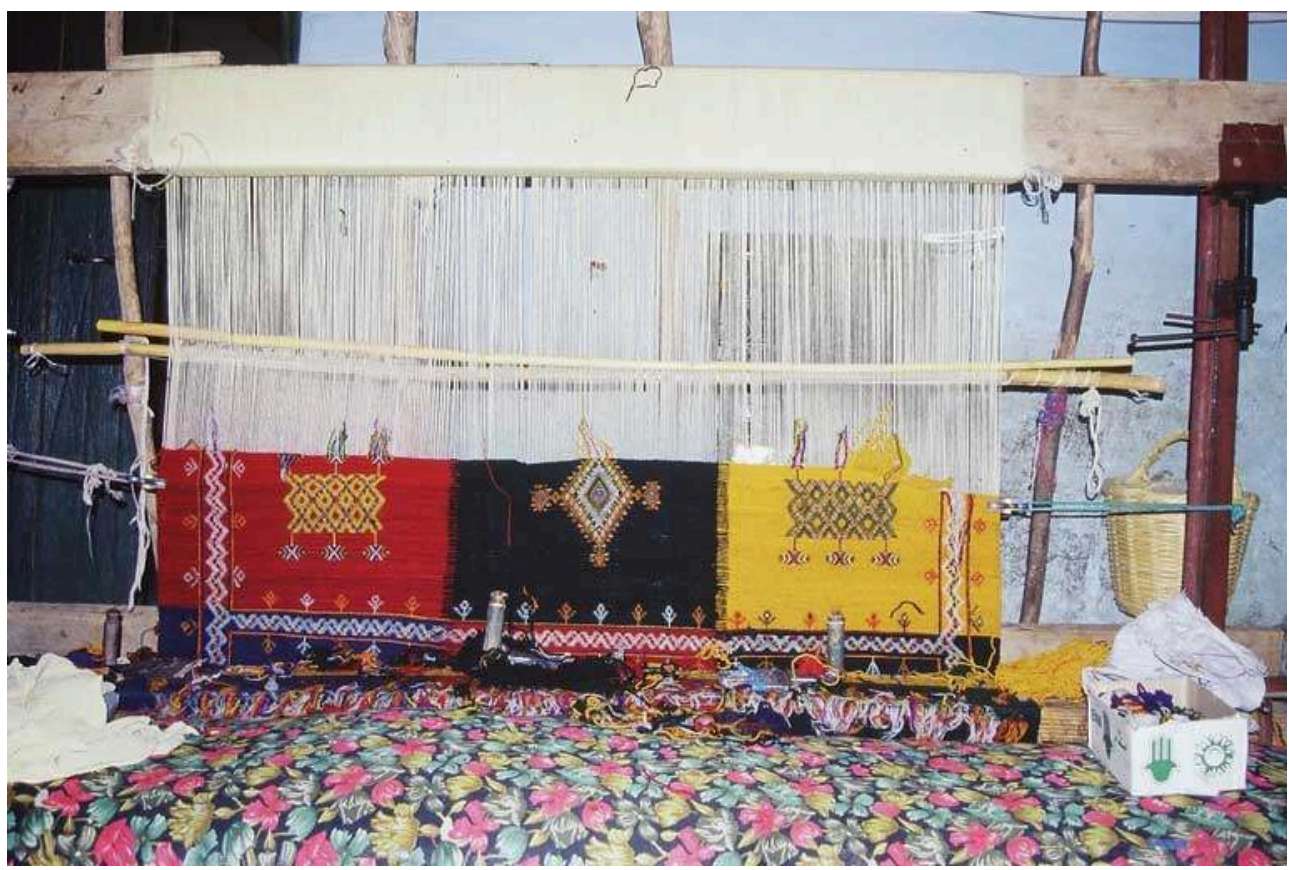

(C)Myriem Naji 
Petit tapis en échiquier à trois couleurs

Les fils indépendants ont été soulevés pour que les motifs inachevés soient plus visibles. Dans les parties jaune et bleue la tisseuse utilise la technique du isghayn. (Fig. 11)

L'une des propriétés des fils est qu'ils forment des unités distinctes les unes des autres par leur couleur et leur épaisseur, des affordances qui signifient qu'ils sont susceptibles d'être classés, alignés rythmiquement ou corrélés pour obtenir des formes géométriques. Une grande variété de motifs peut être obtenue à partir de la combinaison de lignes obliques, parallèles /// ou se rencontrant en $V$ (ou leur contraire $\bigwedge$ ). La juxtaposition de ces formes produit aussi des chevrons horizontalement $\triangle \bigwedge \bigwedge$ et verticalement. Au rhombe, qui se présente sous plusieurs formes (pleines, vides, constituées d'un ou plusieurs rhombes) peuvent être ajoutées d'autres formes, y compris des « indentations multiples » et " motifs digits ciliés, pectinés » (Berque $1964: 17$ ). Avec pratique et répétition, les praticiennes apprennent kinesthésiquement, tactilement et de manière imagée ce qui est commun à toutes les lignes. Elles acquièrent la capacité d'abstraire les traits principaux du concept de ligne et ses concepts associés de lignes parallèles, perpendiculaires et se croisant à différents degrés, pour élaborer des configurations complexes. La symétrie n'est qu'un élément d'une esthétique basée sur une répétition subtile de formes similaires et apparentées (Fig. 4 et 8).

23 Au niveau des motifs, les fils indépendants de décoration forment une succession de lignes qui sont reliées par des nœuds dans leur progression ascendante. Les nœuds sont les points d'articulation où les lignes changent de direction. Le résultat de ces changements de direction qui apparaissent comme des $\backslash$ (iqn) et des $\wedge$ (irzm) sont décrits par les tisseuses comme des ouvertures ou fermetures respectivement. Le motif constitué de ces éléments placés côte à côte de manière horizontale $<>>>>$ ou verticale se nomme arzem-atitqant [ouvre-ferme]. Cette conception d'un espace qui s'ouvre ou se ferme relève de l'expérience sensorimotrice de l'activité technique elle-même. Les gestes du tissage impliquent l'action de passer des fils sur, autour, derrière d'autres, de tirer la trame à l'intérieur d'un passage et de l'en faire ressortir (Fig. 12). 


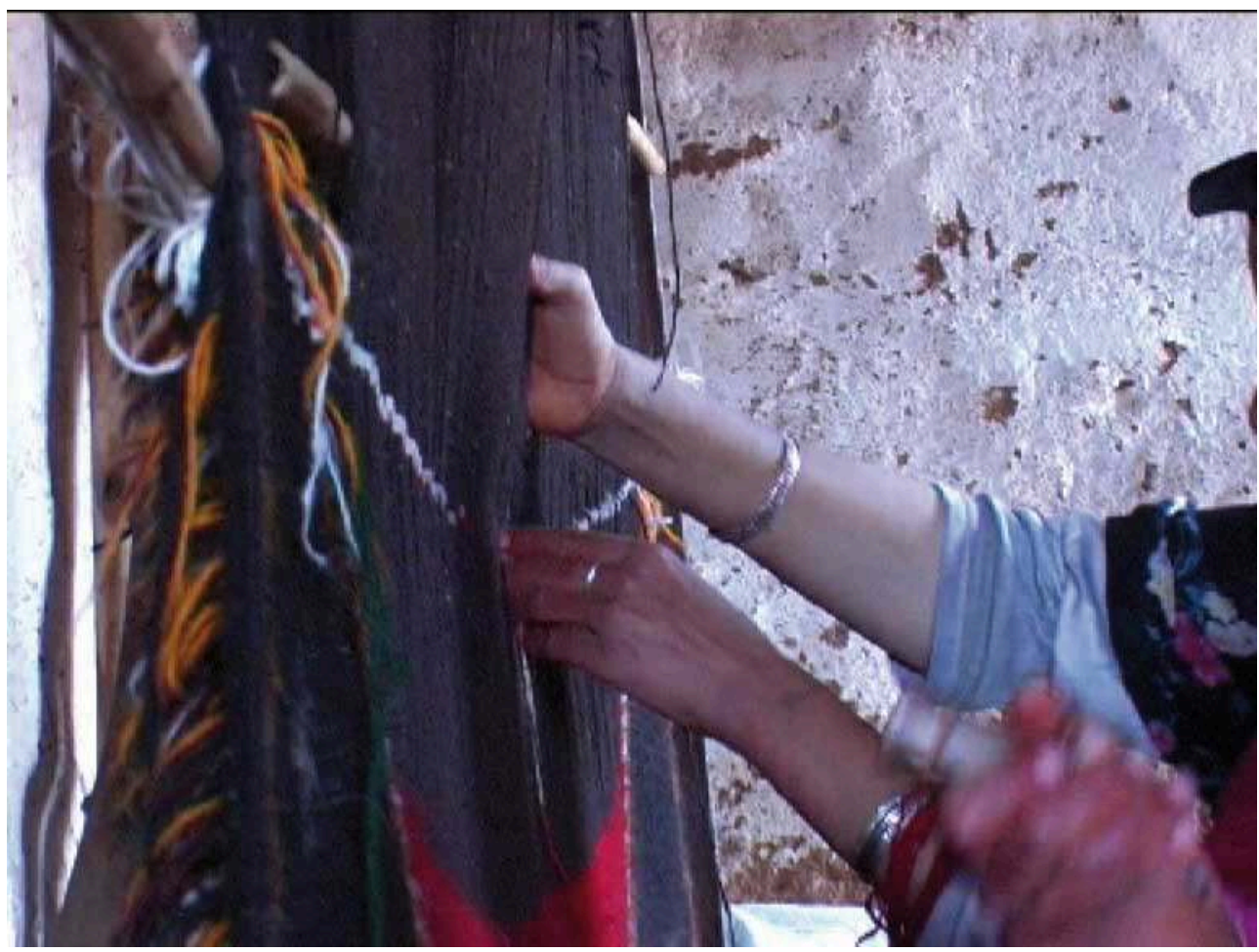

(CMyriem Naji

\section{Passage de trame}

Ici les mains se noient entre les nappes de la chaîne. Une partie des fils des motifs brochés, jetés à l'arrière, apparaissent hirsutes et jaunes sur le dos du tissage. (Fig. 12.)

Cette perception de pénétrer une matière-récipient apparaît aussi dans le vocabulaire utilisé par les tisseuses en situation d'apprentissage. Elles parlent à la débutante en termes de direction (à droite, à gauche, derrière) et usent de verbes de mouvement (skshm! [entre !], sfgh! [sors !], zri! [passe!]). Ces expressions traduisent une expérience physique de déplacement dans l'espace pour atteindre un but similaire à celle identifiée par Lakoff et Johnson (1980 ; Johnson 1987) comme un chemin (path) ou un schéma source-chemin-but.

De plus, dans la culture berbère et arabe (Doutté 1909 ; Westermarck 1926), l'acte de nouer et son résultat sont conçus comme des actions de fermeture aussi bien dans la pratique technique que magique. La signification de l'action de nouer comme une fermeture n'est pas à chercher dans les termes utilisés pour décrire les motifs (Daugherty 2004) mais plutôt dans l'exécution des gestes techniques ${ }^{33}$ qui consistent à enrouler un fil ou un autre matériau autour d'un support et à le serrer de manière plus ou moins lâche et définitive. En fermant avec un lien, en entourant un membre ou le cou et en serrant, on coupe la circulation du sang, le souffle, voire la vie, et ce, pas seulement de façon symbolique. Le nœud est utilisé au quotidien : pour attacher les foulards et ceintures, dans la confection de nouets de protection ou de baluchons servant à porter le linge à la fontaine ou à protéger un casse-croûte de la poussière et des mouches. Le transport de fagots sur le dos ou le placement d'énormes sacs d'orge fraîchement moissonné sur le dos d'un âne requiert de connaître l'art de nouer avec des cordes. 
Le concept de ligne permet de penser le mouvement des fils, leur direction, leur couleur et la forme tracée par leur trajectoire comme un tout. Par exemple, la couleur d'un fils est choisie par rapport au fond sur lequel il sera placé et par rapport aux autres fils du motif. La recherche de contraste entre les couleurs n'est pas seulement d'origine physiologique ou neurologique mais aussi culturelle : les couleurs pastel et le gris ne sont envisageables ni comme fond ni comme motif. Mon choix du jaune paille et du bleu-gris pour les fils décoratifs et du marron et beige pour les fonds a été amplement critiqué par mes amies tisseuses, qui, après avoir tenté en vain de me convaincre d'abandonner des couleurs si démodées pour le fond, n'ont cessé de me recommander de l'orner de motifs rouge foncé ou orange vif. D'autres paramètres, techniques et sociaux interviennent dans la perception. L'accès aux teintures artificielles en offrant une palette de couleur plus variée a étendu les possibilités de combinaison des formes et permis l'élaboration de motifs plus complexes et disposés dans l'espace de manière plus variée que dans le passé. Il a modifié non seulement les possibilités de perception et d'action, mais aussi de pensée, et donc la variété des types de tapis. L'intervention des marchands et la compétition entre les tisseuses ont aussi stimulé la créativité de celles-ci.

\section{Organisation spatiale et mathématique}

Que le tissage soit du domaine des mathématiques (Gerdes 1998 ; Küchler 2001 ; Urton 1997) n'est pas facilement admis par les tisseuses qui assimilent le savoir mathématique à l'éducation formelle. Cependant, elles insistent toutes sur le fait que le calcul (l-ḥsâb) est une propriété centrale de l'activité. Ainsi, Lala Anaya, à Ifanwan, décrit comment sa mère était capable, à la simple vue d'un motif sur un sac de selle tissé, de savoir quels calculs étaient nécessaires pour le reconstituer.

Le tissage est basé sur l'organisation spatiale systématique des fils-lignes. Les gestes de tissage comptent, séparent, classent et mettent les fils en ordre. La faculté cognitive de compter est directement liée au fait que nous avons des doigts que nous pouvons bouger et énumérer (Butterworth 2000) et que nous possédons des mécanismes mathématiques innés comme celui d'isoler immédiatement jusqu'à trois unités (Dehaene 1997). Toutes les opérations du tissage impliquent des gestes, une temporalité et une spatialité liés au nombre. Durant l'ourdissage (Fig. 7), l'espace entre les deux piquets (taggust, pl. tiggusin) qui portent la chaîne doit correspondre à la longueur (tiddi) désirée du tapis, plus une coudée (ighil) par mesure de sécurité. Pour la largeur du tapis, elles mesurent à l'avance les fils qui serviront à construire la chaînette nouée (tigrut) avant de procéder à son exécution (Fig. 13 a et 13 b). Les fils de la chaine sont pairs et impairs. Le même nombre de fils de trame est passé et tassé rythmiquement à l'aide du peigne. 


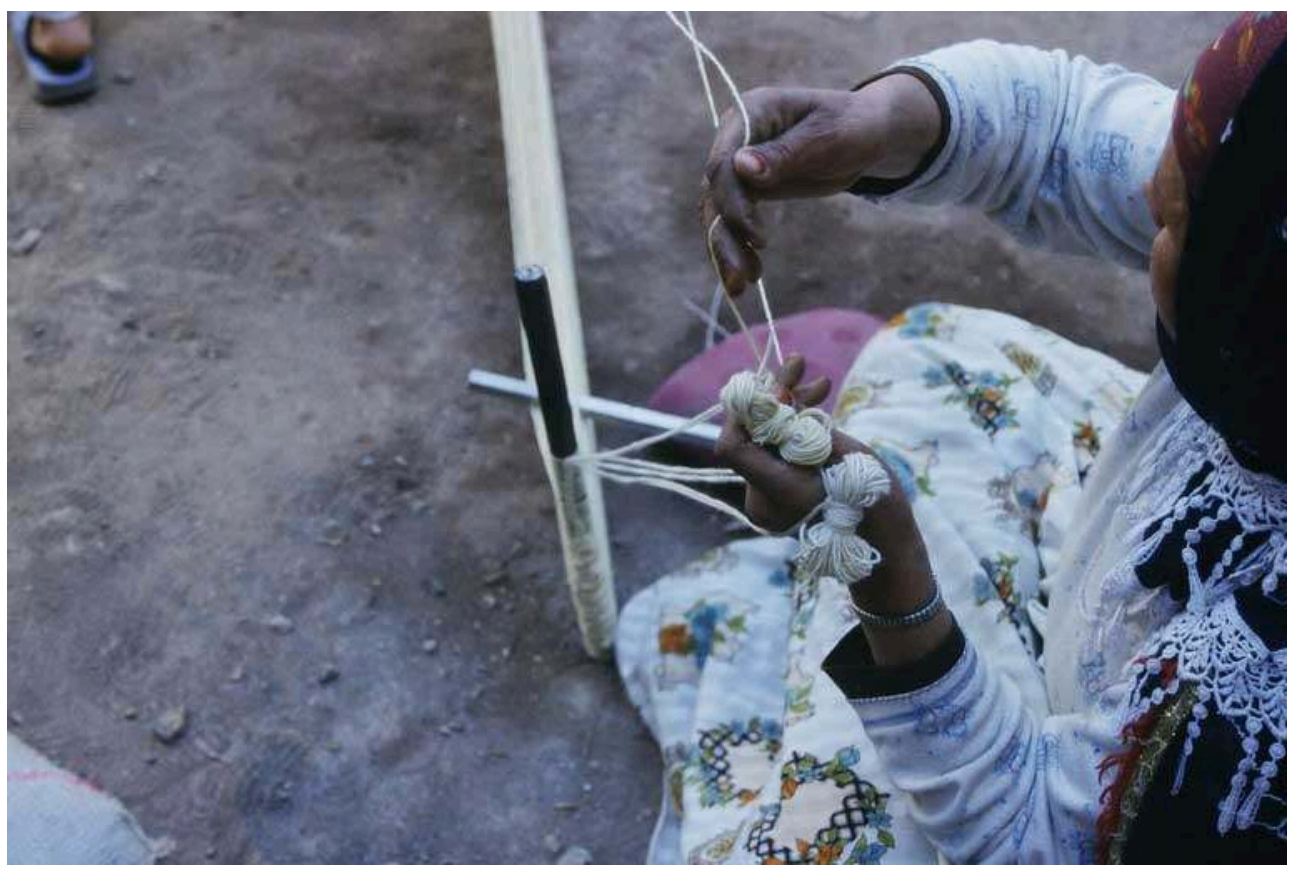

(C)Myriem Naji

\section{Ourdissage, détail d'un tigrut}

Constitution des deux chaînettes (tigrut) qui seront fixées sur les ensouples. Chaque ourdisseuse tient dans une main les deux pelotes de fil (dont la longueur correspond à la largeur du tapis) entre lesquelles elle glisse de l'autre main le fil que lui tend celle qui va et vient entre les deux piquets. (Fig. 13 a et b)

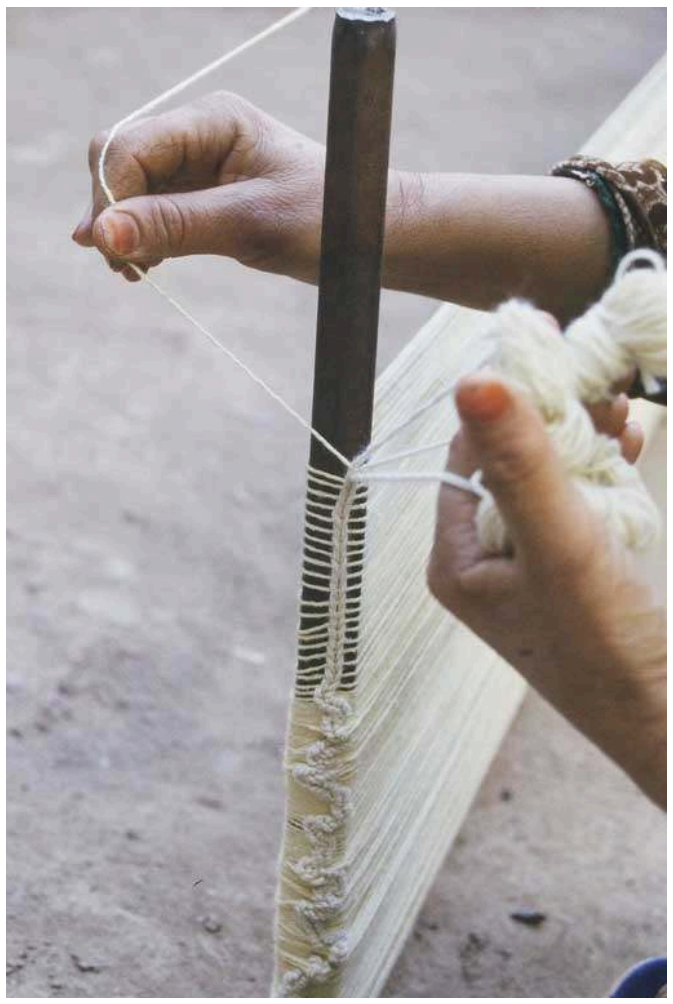

(CMyriem Naji 
Ourdissage, détail d'un tigrut

Constitution des deux chaînettes (tigrut) qui seront fixées sur les ensouples. Chaque ourdisseuse tient dans une main les deux pelotes de fil (dont la longueur correspond à la largeur du tapis) entre lesquelles elle glisse de l'autre main le fil que lui tend celle qui va et vient entre les deux piquets. (Fig. 13 a et b) mesurer leur tapis qui atteignent jusqu'à 8 coudées (environ 4 mètres) de long. Pour les petites mesures, les tisseuses utilisent des unités de mesure fondées sur la main. Tikemst (de ikemz, le pouce) ne comprend que la première phalange du doigt. Titzmam correspond aux deux premières sections de l'index (dont l'ongle) et est à peu près la moitié $(4-5 \mathrm{~cm})$ de l'awrum qui correspond à toute la longueur du majeur depuis son extrémité à fin des phalanges (Fig. 14). L'empan (tardast) est le double de l'awrum. Ces mesures anthropométriques correspondent aussi à des normes esthétiques locales quant à la distance adéquate entre les motifs ou entre ceux-ci et les bordures du tapis.

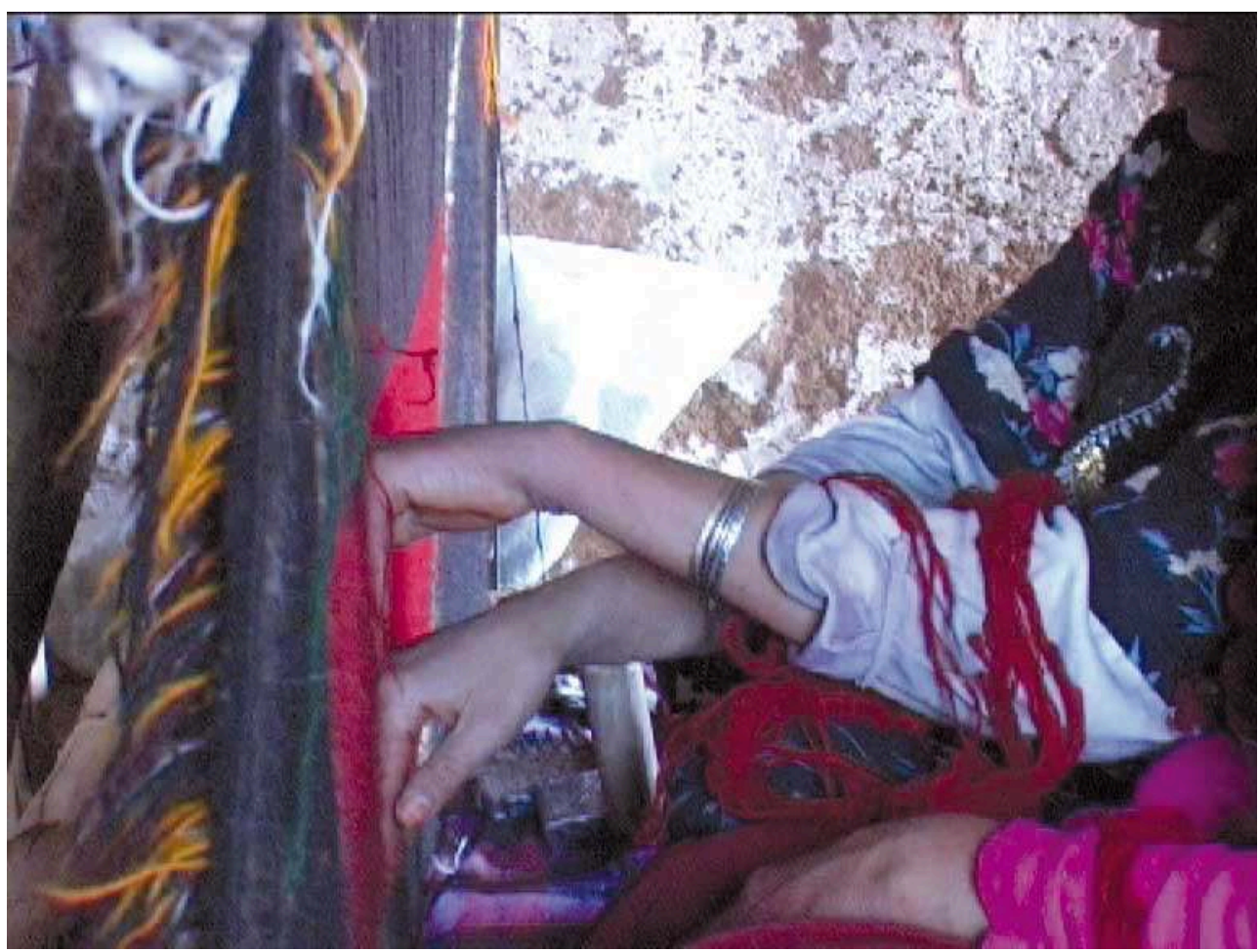

(C)Myriem Naji

Une mesure anthropomorphique

L'awrum (Fig. 14)

La perception seule ne suffit pas à la construction des concepts géométriques (Aebli 1951, in Semadeni $2008: 6$ ) ou mathématiques (Papert 1980) qui sont aussi fondés sur la manipulation physique d'objets et sur des expérimentations par le sujet. Pour Kitcher (1983 : 110-111), la manipulation et la formation d'idées mathématiques abstraites trouvent leur origine dans la manipulation d'objets en deux principales activités : celle d'assembler et celle de corréler. Ranger des objets en groupe implique de les séparer d'autres objets en leur attribuant une position spatiale distincte ; corréler des objets signifie les placer côte à côte, au-dessus ou au-dessous d'autres objets. Selon Lakoff \& Nunez (2000), additionner correspond à «mettre ensemble " ou "compter 
d'avantage " et devient alors synonyme d'« augmenter ». La soustraction devient synonyme d' " enlever » ou de " diminuer ». Multiplier est traduit par la métaphore « il y a beaucoup de » et diviser, par « partager ».

L'addition et la soustraction interviennent dans la construction des motifs, les tisseuses ajoutant (zaydâs) des fils indépendants, et ultérieurement les déduisant (naqsâs) en les « jetant » (garnîs) derrière la chaîne (Fig. 10). Les opérations de multiplication sont moins apparentes aux tisseuses ; elles ont lieu à chaque fois qu'elles ajoutent le même nombre de fils plusieurs fois de part et d'autre d'un axe, ou lorsqu'elles réalisent un motif à échelle inférieure ou supérieure, ce qui implique une diminution proportionnelle du nombre de points.

La division (bțo) est la plus évidente des opérations mathématiques pour les praticiennes. Dès que la chaîne est achevée, elle est marquée au charbon en son milieu. Cependant pour décider de l'emplacement du motif central du tapis, les tisseuses tiennent un compte des coudées au fur et à mesure qu'elles enroulent la partie tissée autour de l'ensouple inférieure (Fig. 3), compte qu'elles déduisent de la longueur totale de la chaîne. La division s'opère aussi lorsque sont posées les fondations, c'est-à-dire les éléments principaux qui organisent la composition. On commence par déterminer le centre du tissage dans sa largueur, puis on subdivise le tapis en portions plus petites, attribuant ainsi une aire de travail à chacune (Fig. 15).

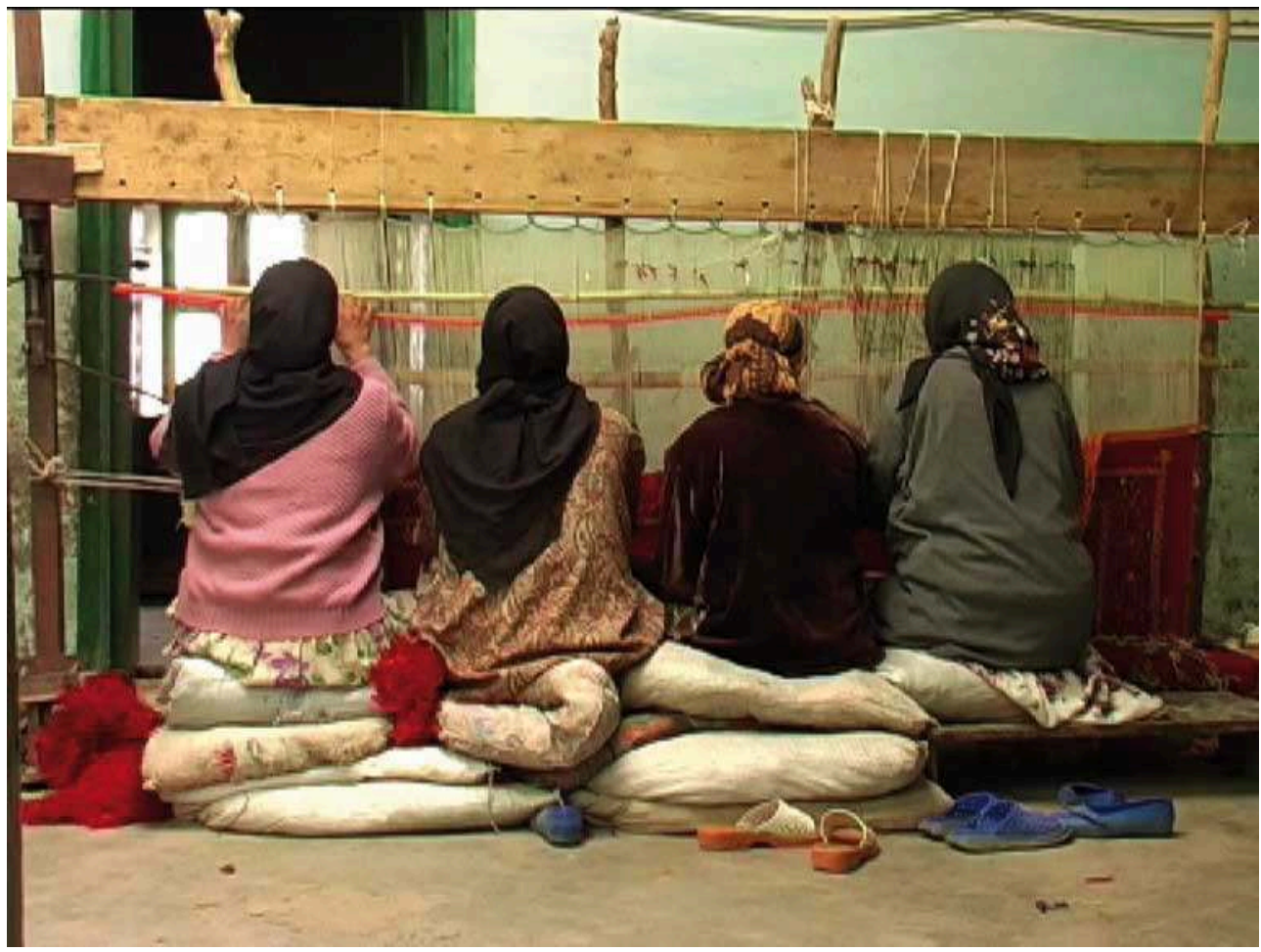

(CMyriem Naji

Organisation spatiale

Les tisseuses se placent côte à côte et chacune se charge de sa partie. À droite, l'emplacement de la cinquième tisseuse, absente est visible. (Fig. 15)

Dans la famille Ayt 'Abdallah l'organisation générale du tapis est discutée la veille des opérations par toutes les tisseuses, leur mère et leur père. Celui-ci informe les tisseuses 
de la dimension et du type de tapis désiré par le marchand. Les tisseuses réfléchissent aux motifs qu'elles désirent produire et négocient leur position respective devant le métier. Pour un tapis de six coudées de large, elles seront cinq à travailler pendant une vingtaine de jours jusqu'à sept heures par jour. Au centre, la plus experte est responsable de l'ensemble et de la cohérence du tapis mais, surtout, du motif central qui coupera le tapis en deux dans sa hauteur déterminant ainsi sa symétrie. Elle s'occupera également de la frise qui forme le cadre du tapis et constitue souvent la ligne de séparation entre les carreaux dans les tapis en échiquier (Fig. 4 et 11). L'organisation spatiale des corps par rapport à la structure en fil aide les tisseuses à maintenir cohérence et symétrie tout au long de l'ouvrage, chacune étant censée se souvenir de ce qu'elle a produit dans la première moitié du tapis. Elle réduit les efforts cognitifs en générant des plans d'action.

33 Au niveau de la composition générale du tapis, l'arrangement symétrique des motifs constitue en même temps une contrainte et une possibilité. La difficulté technique tient en ce qu'un motif donné doit être reproduit à un moment ultérieur sans que son modèle ne soit visible. Ici une autre affordance du fil, qui se prête à être noué de façon temporaire, est exploitée pour aider à la mémorisation : le point central du motif (ou ses extrémités) est marqué par un fil noué lâchement autour du fil de chaîne. Ce repère, déplacé le long du fil de chaîne au fur et à mesure de la progression du tapis, indique un emplacement, une dimension ou une couleur. Dans ce cas, la contrainte physique stimule des opérations cognitives : le fait de devoir tisser du bas vers le haut stimule une capacité à organiser et construire les motifs et concepts ou images de manière ascendante, en plaçant d'abord les éléments extérieurs et centrifuges du motif, lequel a toujours une partie inférieure et supérieure. Avec la pratique, les tisseuses développent une capacité de rotation mentale et de planification qui va de pair avec la recherche de la symétrie.

34 Au niveau de l'exécution du motif, la division intervient aussi : chaque motif est construit symétriquement en plaçant le même nombre de fils de la même couleur à gauche et à droite d'un fil de chaîne (axe) que l'on nomme khît en berbère (« fil » en arabe). La symétrie offre une aide cognitive à la tisseuse qui peut toujours, à partir de la moitié inférieure du motif, reconstituer (même en tâtonnant) la partie supérieure. La pratique répétée de trier et le processus additif de placer les fils de façon ordonnée et dans des directions précises se font en parallèle d'un continuel décorticage mental et physique des motifs en unité-fil (processus de réduction). Par un principe d'accumulation, les opérations mathématiques simples se complexifient vers la création de configurations de plus en plus élaborées. Le stock mémorisé est à son tour enrichi par la manipulation de la matière : la lenteur avec laquelle le tissage progresse encourage l'émergence de nouvelles combinaisons de couleurs ou de formes, qu'elles soient intentionnelles expérimentations ou le produit d'erreurs.

$35 \&$

Le concept de ligne nous permet donc de rendre compte partiellement de la manière dont les tisseuses pensent grâce aux affordances de la matière et de la technique mais aussi de leur corps et s'adaptent aux contraintes structurelles et matérielles du tissage pour organiser et planifier la production de tapis symétriques à motifs géométriques complexes. L'entité géométrique " ligne » est le résultat de la synthèse de multiples expériences sensorimotrices et de multiples incorporations (matière, temps, espace, images, corps des autres) unies par la pratique. Ainsi, les tisseuses aiguisent leurs 
compétences mathématiques et leur capacité d'abstraction, de cognition spatiale et de planification. Elles développent une pensée visuo-spatiale et une mémoire multisensorielle impliquant des éléments verbaux, visuels, auditifs et kinesthésiques leur permettant d'identifier certaines formes et de les reproduire habilement dans une relation dialectique entre geste et image.

Toutes les photos sont de l'auteure, sauf mention contraire.

\section{BIBLIOGRAPHIE}

Aebli, Hans, 1951, Didactique psychologique, application à la didactique de la psychologie de Jean Piaget. Neuchâtel, Delachaux et Niestlé. PUF : 12-24.

Butterworth, Brian 2000, The Mathematical Brain. Londres, Papermac.

Childs, Carla P. \& Greenfield, Patricia. M., 1980, « Informal Modes of learning and teaching : The Case of Zinacanteco weaving ", in Studies in cross-cultural psychology : vol. 2. N. Warren ed., New York, Academic Press : 269-316.

Daugherty, Patricia 2004, « Cognitive Structures and Body metaphors in the Aesthetic and Everyday Discourse of Turkish-Yörük », in D.K. Washburn \& D.W. Crowe eds. , Symmetry cComes of Age : the Role of Pattern in Culture : 04-315.

Dehaene, Stanislas 1997, [ Vs. Fr. 2007, La bosse des maths, Paris Odile Jacob ] The Number Sens : how the Mind Creates Mathematics. New York, Oxford University Press.

Dehaene, Stanislas Doutté, Edmond 1984 [1909], Magie et Religion dans l'Afrique du Nord. Paris, Maisonneuve.

Gallese, V. 2000 a, « The Inner Sense of Action : Agency and Motor Representations », Journal of Consciousness Studies, $7: 23-40$.

Gallese, Vittorio 2000 b, « La mise en phase intentionnelle. Le système miroir et son rôle dans les relations interpersonnelles/Intentional Attunement. The Mirror Neuron System and its Role in Interpersonal Relations ». (Traduit de l'anglais par Anne-Marie Varigault) < http // : www.interdisciplines.org/mirror/papers/1/version/fr>. Consulté le 12 avril 2009.

Gerdes, Paulus, 1998, Women, Art and Geometry in Southern Africa. Trenton, N.J., Africa World Press.

Gibson, James 1979, The Ecological Approach to Visual Perception. Boston (Mass.), Houghton Mifflin.

Jeannerod, Marc, 1994, « The Representing Brain : Neural Correlates of Motor Intention and Imagery ", Behavioral and Brain Sciences, 17 (2) : 187 - 245.

Johnson, Mark, 1987, The Body in the Mind. Chicago, University of Chicago Press.

Johnson, Mark, 1999, « Embodied reason », in G. Weiss \& H. Fern Haber eds., Perspectives on Embodiment : the Intersections of Nature and Culture. Londres, Routledge : 81-102. 
Keller, Charles M. \& Keller, Janet D. , 1996, Cognition and Tool Use : the Blacksmith at Work. Cambridge, Cambridge University Press.

Kirsch, David, 1995, The Intelligent Use of Space, Artificial Intelligence 73 :31-68.

Kitcher, Philip, 1984, The Nature of Mathematical Knowledge. Oxford, Oxford University Press.

Knappett, Carl, 2004, «The Affordances of Things : a Oost-Gibsonian Perspective on the Relationality of Mind and Matter ». in Rethinking Materiality : The Engagement of Mind with the Material World. E. DeMarrais, C. Gosden \& C. Renfrew eds., McDonald Institute for Archaeological : 43-51.

Küchler, Susanne, 2001, "Why knot ? A Theory of Art and Mathematics », in Beyond Aesthetics : Art and Technologies of Enchantment. C. Pinnery \& N.Thomas eds., Oxford and New York, Berg, 57-79.

Lakoff, George \& Mark Johnson, 1980, Metaphors we Live by Chicago, University of Chicago Press.

Lakoff, George \& Rafael E. Nùñez, 2000, Where Mathematics Comes From : How the Embodied Mind Brings Mathematics into Being. New York, Basic Books.

Lave, Jean \& Wenger, Étienne, 1991, Situated Learning : Legitimate Peripheral Participation. Cambridge, Cambridge University Press.

Lave, Jean, 1988, Cognition in Practice : Mind, Mathematics and Culture in Everyday Life. Cambridge, Cambridge University Press.

Leroi-Gourhan, Andre, 1943, Évolution et Techniques. L'Homme et la Matière. Paris, Albin Michel. Leroi-Gourhan, Andre, 1945, Évolution et Techniques. Milieu et Techniques. Paris, Albin Michel.

Marchand, Trevor H.J. 2007. "Crafting Knowledge : The Role of "Parsing and Production" in the communication of skill-based knowledge among masons ", in M. Harris ed., Ways of Knowing. Oxford, Berghahn, 173 - 193.

Marchand, Trevor H.J., 2008, « Muscles, Morals and Mind : Craft Apprenticeship and the Formation of Person », British Journal of Educational Studies, 56 (3) : 245 - 271.

Merleau-Ponty, Maurice, 1945, Phénoménologie de la perception. Paris, Gallimard.

Naji, Myriem, 2009, « La Formation de féminités à travers le tissage dans le Sirwa (Maroc) ». in C. Rosselin \& M.-P. Julien eds., Le Sujet contre les objets... tout contre : ethnographies de cultures matérielles. Paris, Éditions du CTHS : 243-263.

Norman, Donald A., 1999, Affordances, Conventions and Design. Interactions, $6: 38-43$.

Papert, Seymour, 1980, Mindstorms : children, computers, and powerful ideas. New York, Basic Books.

Quéré, Louis. 1997, « La situation toujours négligée ? ", Réseaux 15 ,n 85 : 163-192.

Rosselin, Celine, 2006, "Incorporation ", in A. Bernard ed., Dictionnaire du corps en sciences humaines et sociales. Paris, CNRS.

Urton, Gary, 1997, The Social Life of Numbers : a Quecha Ontology of Numbers and Philosophy of Arithmetic. Austin, University of Texas Press.

Varela, Francisco J., Evan Thompson \& Eleanor Rosch ed., 1991. [Vs. Fr. 1999, L’Inscription corporelle de l'esprit. Paris, Seuil The Embodied Mind : Cognitive Science and Human Experience. Londres, MIT Press. 
Semadeni, Zbigniew, 2008, « Deep Intuition asa Level in the Development of the Concept

Image ", Educational Studies in Mathematics, vol. $68: 1-17$.

Westermarck, Edward, 1926, Ritual and Belief in Morocco. Londres, Macmillan.

\section{NOTES}

1. Chez les Ayt Ubiâl et Ayt Waghrda, seules les femmes mariées tordent leurs cheveux en des sortes d'anglaises qu'elles portent comme deux nattes sous leurs foulards.

2. Le terme Berber astta désigne à la fois le tissage comme objet-en-devenir et activité, et les instruments techniques.

3. De même, la plupart des chercheurs anglosaxons en ethnomathématiques analysent l'objet fini (tapis, panier, cheveux tressés) plutôt que les praticiens à l'œuvre sur un objet-en-devenir.

4. La coudée est utilisée par les marchands sur le souk lorsqu'ils mesurent la taille des tapis pour évaluer son coût en matière première et donc sa valeur.

\section{RÉSUMÉS}

Le Fil de la pensée tisserande. "Affordances" de la matière et des corps dans le tissage. Cet article tente de répondre à la question : comment les tisseuses du Sirwa (Maroc) parviennent à produire des tapis symétriques sans l'usage de supports cognitifs tels que des tapis ou des schémas en papier?

La notion d'affordance (appliquée ici à la matière, à l'organisme et au cerveau) et la perspective des théories de l'embodiment ou de la cognition située nous permettent d'examiner les relations entre les praticiennes et leur environnement à travers la médiation du corps. À partir du concept de "ligne ", nous explorons les formes de savoir que la technique du tissage stimule : compétences mathématiques, capacité d'abstraction, de mémorisation, de cognition spatiale et de planification.

Weaving the Thread of Thought. Material and Bodily Affordances in Southern Morocco. This article attempts to answer the following question : how do weavers in the Sirwa, south Morocco, manage to produce symmetrical carpets without the use of any cognitive supports ? I use the notion of affordance (applied here to matter, the body and the brain), an embodiment approach and the situated action theory to examine the relationship between practitioners and their environment through the mediation of their body. Using the « line » concept I explore the forms of knowledge that are stimulated by the practice of weaving : mathematical skills, abstraction, memorisation, spatial cognition and planning.

\section{INDEX}

Keywords : affordance, body, cognition, embodiment, geometry, knots, mathematics, weaving Mots-clés : affordance, cognition, corps, embodiment, géométrie, mathématiques, nœud, tissage 